Article

\title{
Synthesis, Characterization and Biological Studies of Some Novel Thieno[2,3-d]pyrimidines
}

\author{
Khulud M. Al-Taisan ${ }^{1, *}$, Hassan M. A. Al-Hazimi ${ }^{2}$ and Shar S. Al-Shihry ${ }^{1}$
}

1 Chemistry Department, College of Science, King Faisal University, P.O. Box 400, Hofuf 31982, Saudi Arabia

2 Chemistry Department, College of Science, King Saud University, P.O. Box 2455, Riyadh 11451, Saudi Arabia

* Author to whom correspondence should be addressed; E-Mail: Kaltisan@yahoo.com.

Received: 29 March 2010; in revised form: 20 May 2010 / Accepted: 27 May 2010 /

Published: 1 June 2010

\begin{abstract}
Several 2-unsubstituted thieno[2,3-d]pyrimidines have been prepared from 2aminothiophene-3-carboxylic acid esters and their carbonitrile analogs. Some triazolothienopyrimidine and 2-thioxothienopyrimidine representatives have also been synthesized using thermal and microwave (MW) irradiation techniques. Structures of the prepared compounds were elucidated on the basis of IR, NMR, 2D NMR and mass spectral data. The biological activity of some selected synthesized compounds was also examined.
\end{abstract}

Keywords: thienopyridines; triazolothienopyrimidines; antibacterial activity; cytotoxic activity

\section{Introduction}

Heterocycles containing the thienopyrimidine moiety (Figure 1) are of interest because of their interesting pharmacological and biological activities [1-6]. Thus, over the last two decades many thienopyrimidines have been found to exhibit a variety of pronounced activities, for example, as antiinflammatory [3,7], antimicrobial [3,8], antiviral [9] and analgesic [7,10] agents. Some thienopyrimidine derivatives showed good antitumor activity [11], while compounds with the general structure designated by E (Figure 1) showed potent and specific cytotoxicity against several leukemia cell lines [4]. Motivated by the aforementioned biological and pharmacological importance of the title 
compounds, and as continuation with our previous work on thienopyrimidines [12,13], we report herein the synthesis of some new heterocycles incorporating a thienopyrimidine moiety. Representative compounds among the synthesized thienopyrimidines were tested and evaluated as antibacterial agents and for cytotoxicity against some cancer cell lines.

Figure 1. Some biologically active thienopyrimidines.

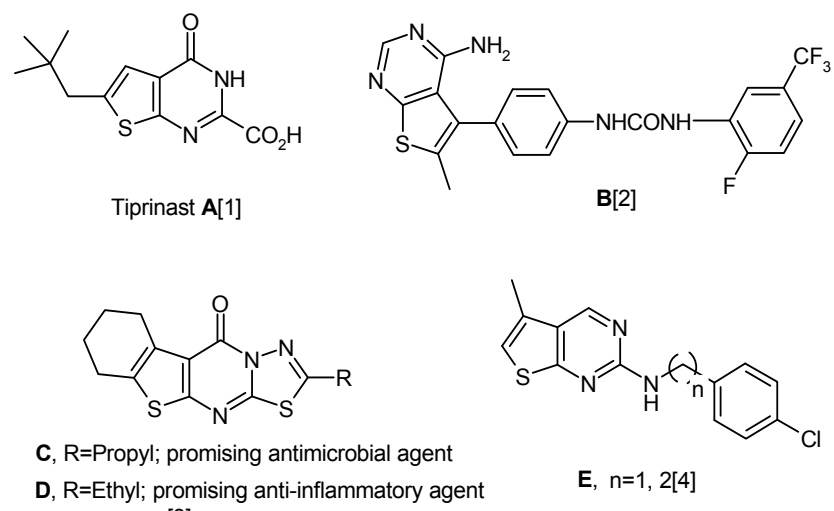

[3]

\section{Results and Discussion}

\subsection{Chemistry}

The synthetic pathways depicted in Schemes 1-4 outline the chemistry of the present study. Thus, the 2-amino-3-thiophene carboxylic ester starting materials 1a-d and 32a,b are easily prepared following the well established procedure reported in the literature [14,15]. Treatment of 1a,b with $\mathrm{ClCO}\left(\mathrm{CH}_{2}\right)_{4} \mathrm{Cl}$ gave the corresponding amides $\mathbf{2 a , b}$, which in turn gave compounds $\mathbf{3 a}, \mathbf{b}$ upon refluxing in DMF with 1-(methoxyphenyl)piperazine in the presence of potassium carbonate (Scheme 1). The IR spectra of $\mathbf{2 a , b}$ and 3a,b showed two peaks in the $1,675-1,678 \mathrm{~cm}^{-1}$ and $1,658-1,662 \mathrm{~cm}^{-1}$ range corresponding to ester and amide carbonyl absorptions, respectively. The structure of the unpreviously unknown $\mathbf{2 a , b}$ and 3a,b were unambiguously assigned on the basis of their NMR and MS data (see Experimental). Reaction of $\mathbf{3 a}, \mathbf{b}$ with hydrazine in hot methanol led to cyclization to the corresponding thienopyrimidines in moderate yields. The IR spectra of $\mathbf{4 a}, \mathbf{b}$ exhibited a strong peak corresponding to the carbonyl group at $1,672-1,680 \mathrm{~cm}^{-1}$. The ${ }^{1} \mathrm{H}-\mathrm{NMR}$ spectrum of $\mathbf{4 a}$ in $\mathrm{CDCl}_{3}$ showed a broad singlet at $\delta 4.96\left(\mathrm{NH}_{2}\right)$, a methoxyl proton singlet and four aromatic proton resonances (Table 1). This spectrum also showed the presence of the cyclohexane and piperazine ring methylene protons as well as the butyl moiety, as judged from the DEPT spectrum. The mass spectrum of 4a showed a prominent molecular ion peak $\left[\mathrm{M}^{+}\right]$as the base peak and a fragmentation pattern consistent with its structure. The ${ }^{13} \mathrm{C}-\mathrm{NMR}$, combined with the DEPT spectrum of $\mathbf{4 a}$, confirmed the existence of 25 carbons, including a benzene ring, 12 methylene carbons (10 signals, taking in consideration the equivalency of methylene protons in the piperazine ring), methoxyl and thienopyrimidine carbons. HMBC spectroscopy (Table 1) were used to elucidate the structure and to establish the complete NMR assignments of 4a. Starting from $\mathrm{H}^{2}{ }^{\prime}$ the most shielded aromatic proton, showed two-bond correlations with $\mathrm{C}-1^{\prime}$ and $\mathrm{C}-3^{\prime}{ }^{\prime}$, and showed three-bond correlations with $\mathrm{C}-4^{`}$ and $\mathrm{C}-6^{\prime} . \mathrm{H}-5^{`}$ and 
methoxyl protons showed three-bond correlations with $\mathrm{C}-1$ '. Further, $\mathrm{H}-5^{`}$ and $\mathrm{C}-\mathrm{a}$ ' of the piperazine ring showed respectively two-bond and three-bond correlations to C-6 .

Scheme 1. Synthesis of compound 2-4.

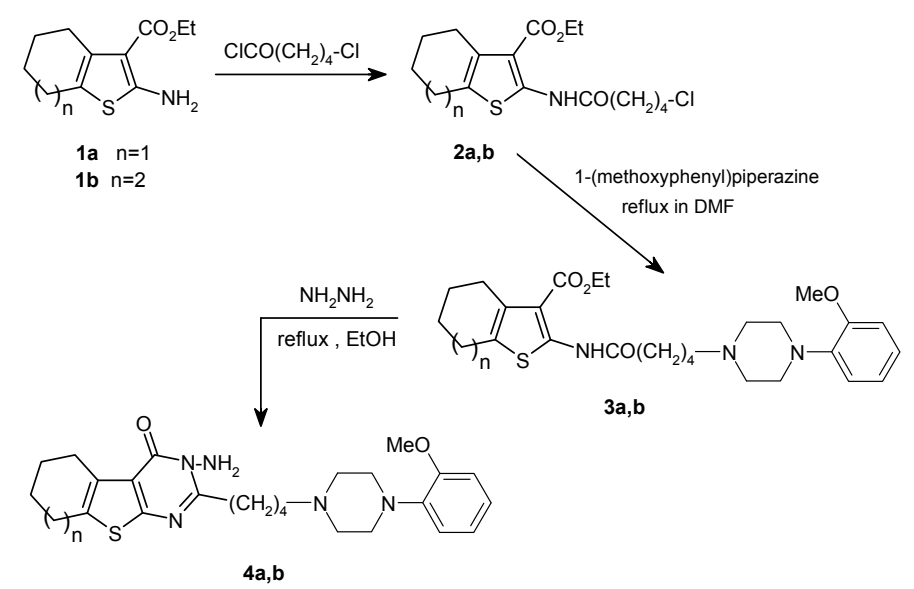

Table 1. ${ }^{1} \mathrm{H}-\mathrm{NMR},{ }^{13} \mathrm{C}-\mathrm{NMR}$ and $\mathrm{HMBC}$ correlations of $\mathbf{4 a}\left(\mathrm{CDCl}_{3}\right)$.<smiles>COc1ccccc1N1CCN(C[C+]=CCCc2nc3sc4c(c3c(=O)n2N)CCCC4)CC1</smiles>

\begin{tabular}{|c|c|c|c|c|}
\hline \multirow[t]{2}{*}{ No } & \multirow{2}{*}{$\frac{13 \text {-NMR(DEPT) }}{\delta_{\mathrm{C}}}$} & \multirow{2}{*}{$\begin{array}{c}{ }^{1} \mathbf{H}-\mathbf{N M R} \\
\delta_{\mathrm{H}} \\
\end{array}$} & \multicolumn{2}{|c|}{ HMBC } \\
\hline & & & ${ }^{2} J$ & ${ }^{3} J$ \\
\hline 1 & - & & & \\
\hline 2 & $156.97(\mathrm{C})$ & & & \\
\hline 3 & - & $4.96\left(\mathrm{NH}_{2}\right)$ & & $\mathrm{C}-2$ \\
\hline 4 & $161.57(\mathrm{C})$ & & & \\
\hline 5 & $158.18(\mathrm{C})$ & & & \\
\hline 6 & $130.91(\mathrm{C})$ & & & \\
\hline 7 & $119.87(\mathrm{C})$ & & & \\
\hline 8 & $141.34(\mathrm{C})$ & & & \\
\hline 9 & $25.16\left(\mathrm{CH}_{2}\right)$ & 2.71 & $\mathrm{C}-6,10$ & $\mathrm{C}-5,7,11$ \\
\hline 10 & $22.24\left(\mathrm{CH}_{2}\right)$ & 1.83 & C-9, 11 & C- 6,12 \\
\hline 11 & $22.95\left(\mathrm{CH}_{2}\right)$ & 1.83 & C-10, 12 & C-7, 9 \\
\hline 12 & $25.39\left(\mathrm{CH}_{2}\right)$ & 2.95 & $\mathrm{C}-7,11$ & $C-6,10$ \\
\hline $1^{\prime}$ & $152.29(\mathrm{C})$ & & & \\
\hline $2^{\prime}$ & $111.31(\mathrm{CH})$ & $6.83(\mathrm{~d})^{*}$ & C-1', 3' & C-4', 6' \\
\hline $3^{\prime}$ & $121.01(\mathrm{CH})$ & $6.88-6.93$ & C-2', 4' & C-1', 5' \\
\hline $4^{\prime}$ & $122.84(\mathrm{CH})$ & $6.97(\mathrm{t})^{*}$ & C-3', 5' & C-2', 6' \\
\hline $5^{\prime}$ & $118.22(\mathrm{CH})$ & $6.88-6.93$ & C-4', 6' & C-1', 3' \\
\hline $6^{\prime}$ & $133.05(\mathrm{C})$ & & & \\
\hline $\mathrm{O}-\mathrm{CH}_{3}$ & $55.34\left(\mathrm{CH}_{3}\right)$ & 3.84 & & $\mathrm{C}-1^{\prime}$ \\
\hline 1" & $33.99\left(\mathrm{CH}_{2}\right)$ & $2.98(\mathrm{t})$ & C-2", 2 & C-3" \\
\hline 2" & $26.25\left(\mathrm{CH}_{2}\right)$ & 1.65 & C-1", 3" & C-2, 4" \\
\hline 3" & $24.65\left(\mathrm{CH}_{2}\right)$ & 1.83 & C-2", 4" & C-1" \\
\hline 4" & $58.32\left(\mathrm{CH}_{2}\right)$ & $2.47(\mathrm{t})$ & C-3" & C-a, 2" \\
\hline $\mathbf{a}$ & $53.75\left(2 \mathrm{CH}_{2}\right)$ & $2.66\left(2 \mathrm{CH}_{2}\right)$ & $\mathrm{C}-\mathbf{a}^{\prime}$ & $\mathrm{C}-4^{\prime \prime}$ \\
\hline $\mathbf{a}^{\prime}$ & $50.33\left(2 \mathrm{CH}_{2}\right)$ & $3.09\left(2 \mathrm{CH}_{2}\right)$ & C-a & $\mathrm{C}-6$ \\
\hline
\end{tabular}

$* J=7.0 \mathrm{~Hz}$. 
A two bond-correlation observed from a $-\mathrm{CH}_{2}$ to $\mathrm{C}-\mathrm{a}$, established the assignments of the chemical shifts of the piperazine ring methylene groups. $\mathrm{H}-1^{\text {" }}$ and the amino protons showed two-bond and three-bond correlations with $\mathrm{C}-2$, respectively. The three-bond correlation from a- $\mathrm{CH}_{2}$ to $\mathrm{C}-4$ " assigned the chemical shifts of the butyl protons in compound 4a. On the other hand, the 9- $\mathrm{CH}_{2}$ showed three-bond correlations with C-5 and C-7 and two-bond correlations with C-6 and C-10, while 12- $\mathrm{CH}_{2}$ exhibited three-bond correlation to $\mathrm{C}-6$ and two-bond correlations to $\mathrm{C}-7$ and $\mathrm{C}-11$ carbons. These correlations unequivocally confirmed the assignments of all carbons of the cyclohexane and thiophene rings. The signal at $\delta_{\mathrm{C}} 141.34$ must correspond to $\mathrm{C}-8$ since this carbon has no correlation with any protons in the HMBC spectrum of $\mathbf{4 a}$.The mass spectrum of $\mathbf{4 b}$ exhibited a molecular ion peak at $\mathrm{m} / z$ 481. Its NMR spectral data are similar to the corresponding data of $\mathbf{4 a}$ with an additional $\delta_{\mathrm{H}}$ at 1.82 and $\delta_{\mathrm{C}}$ at 23.11 for the methylene group.

In another pathway, thienopyrimidine dione $\mathbf{6}$ and the 2-unsubstituted-thienopyrimidines 7-9 have been synthesized as depicted in Scheme 2. The structures of 6-8 were elucidated on the basis of their various spectral data (see Experimental), including their mass spectral data which were consistent with the proposed structures. Compound $\mathbf{8}$ failed to give the condensation product $\mathbf{9}$. None of the thienopyrimidines in Scheme 2 were previously prepared, with the exception of 7a,b [13]. The ${ }^{1} \mathrm{H}$ NMR spectrum of 8a revealed two singlets at $\delta 7.62$ and $\delta 13.14$, each integrating for one proton, corresponding to the protons at position 2 and for the $\mathrm{NH}$, respectively. Compounds 11a,c,d were synthesized from 10 upon treatment with sodium ethoxide. The IR spectra of 11a,c,d are characterized by two bands in the $1,664-1,681 \mathrm{~cm}^{-1}$ and $3,157-3,427 \mathrm{~cm}^{-1}$ range due to the $\mathrm{C}=\mathrm{O}$ and $\mathrm{NH}$ stretching frequencies, respectively. These spectra also showed a band in the 1,110-1,174 $\mathrm{cm}^{-1}$ range, which corresponds to the $\mathrm{C}=\mathrm{S}$ absorption in $11 .{ }^{13} \mathrm{C}-\mathrm{NMR}$ spectra of the latter compounds revealed two signals at around $\delta 173$ and 157 which were attributed to the $\mathrm{C}=\mathrm{S}$ and $\mathrm{C}=\mathrm{O}$, respectively, in addition to the other carbon signals at the expected values. Two singlets at $\delta 4.43$ and 4.24 in the ${ }^{1} \mathrm{H}-\mathrm{NMR}$ spectrum of 11d, each integrating for two protons, correspond to the $\mathrm{CH}_{2}$ of the benzyl group and the $\mathrm{CH}_{2}$ at position 2 of the piperidine moiety in the molecule, respectively. This spectrum also displayed other two multiplet signals at $\delta 3.15\left(\mathrm{CH}_{2}\right)$ and $\delta 3.39\left(\mathrm{CH}_{2}\right)$ in addition to the signals of the protons for two $\mathrm{NH}$ groups at $\delta 11.40$ (br s) and $\delta 12.50$.

Compound 11a reacted with hydrazine to give the corresponding hydrazino derivative $\mathbf{1 4}$ which in turn was transformed into the pyrazolothienopyrimidines 15-17 upon treatment with triethyl orthoformate, triethyl orthoacetate and carbon disulphide. A singlet at $\delta 9.01$ in the ${ }^{1} \mathrm{H}-\mathrm{NMR}$ of $\mathbf{1 5}$ corresponds to the proton in the triazole ring, while a singlet at $\delta 2.98$ in the similar spectrum of $\mathbf{1 6}$ corresponds to the methyl substituent in the latter heterocylic ring. Other than that, the data of both spectra were almost identical. Expected ${ }^{13} \mathrm{C}$-NMR chemical shifts for both compounds were observed (see Experimental).

The condensation products $\mathbf{1 2}$ and $\mathbf{1 3}$ were also obtained upon treatment of $\mathbf{1 1 a}$ with some aromatic hydrazides, as elaborated in Scheme 2. Accordingly, cyclization of 11a to the triazolo-derivatives 12 and 13 occured through the $\mathrm{NH}$ at position 1, as shown by the HMBC spectrum of $\mathbf{1 3}$ which indicated a two bond correlation of the hydrogen of $\mathrm{NH}$ at position 3 to the $\mathrm{C}-4$, and in fact this would be the expected case where the latter $\mathrm{NH}$ is an amide. A literature survey revealed that these condensed thienopyrimidines 12-17 have not been previously prepared, and their NMR spectral data were in full agreement with the proposed structures. The assignment of the various carbons in the ${ }^{13} \mathrm{C}-\mathrm{NMR}$ 
spectra of 12-17 was accomplished with the aid of DEPT-135 and HETCOR experiments. The HMBC correlations of 13, helpful in the assignment of chemical shifts, are shown in Figure 2.

Scheme 2. Synthesis of compounds 5-17.<smiles>[R]c1sc2nc[nH]c(=O)c2c1[R]</smiles>

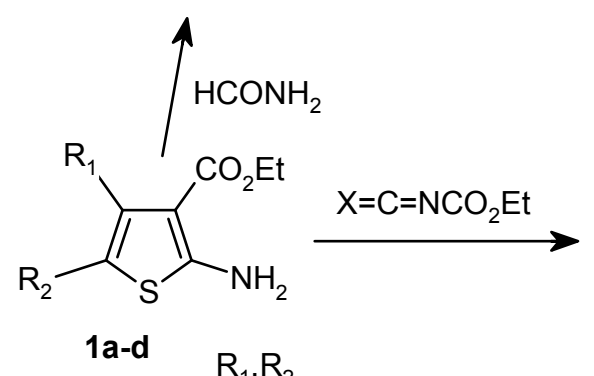

$$
\begin{aligned}
& \quad \frac{\mathrm{R}_{1}, \mathrm{R}_{2}}{\text { a }-\left(\mathrm{CH}_{2}\right)_{4}^{-}} \\
& \text {b }-\left(\mathrm{CH}_{2}\right)_{5^{-}} \\
& \text {c } \mathrm{CH}_{3}, \mathrm{CH}_{3} \\
& \text { d }-\left(\mathrm{CH}_{2}\right)_{2}-\mathrm{N}\left(\mathrm{CH}_{2} \mathrm{Ph}\right)-\mathrm{CH}_{2}-
\end{aligned}
$$<smiles>[R]c1sc(N=[N+]=O)c(C(=O)OCC)c1C(=O)OCC</smiles>

$5, X=\mathrm{O}, \mathrm{b}$<smiles>[R]c1sc2[nH]c(=S)[nH]c(=O)c2c1[R]</smiles><smiles>O=c1c2c3c(sc2nc2[nH]ncn12)CCCC3</smiles><smiles>NNc1nc2sc3c(c2c(=O)[nH]1)CCCC3</smiles><smiles>Cc1n[nH]c2nc3sc4c(c3c(=O)n12)CCCC4</smiles>

16<smiles>O=c1c2c3c(sc2nc2[nH][nH]c(=S)n12)CCCC3</smiles>

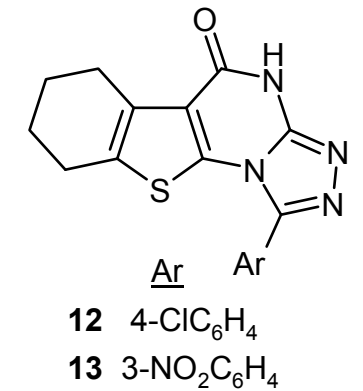

Figure 2. Selected HMBC spectrum of $\mathbf{1 3 .}$

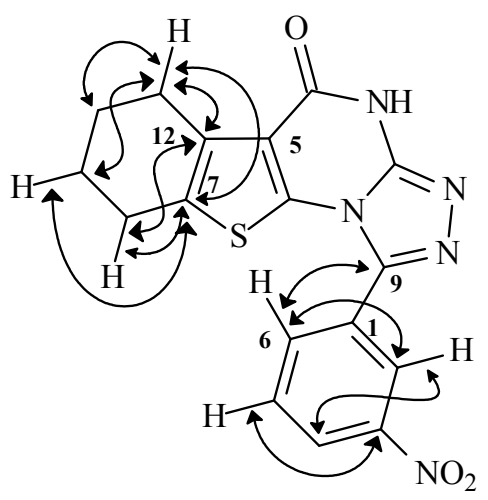


Scheme 3. Synthesis of compounds 18-31.

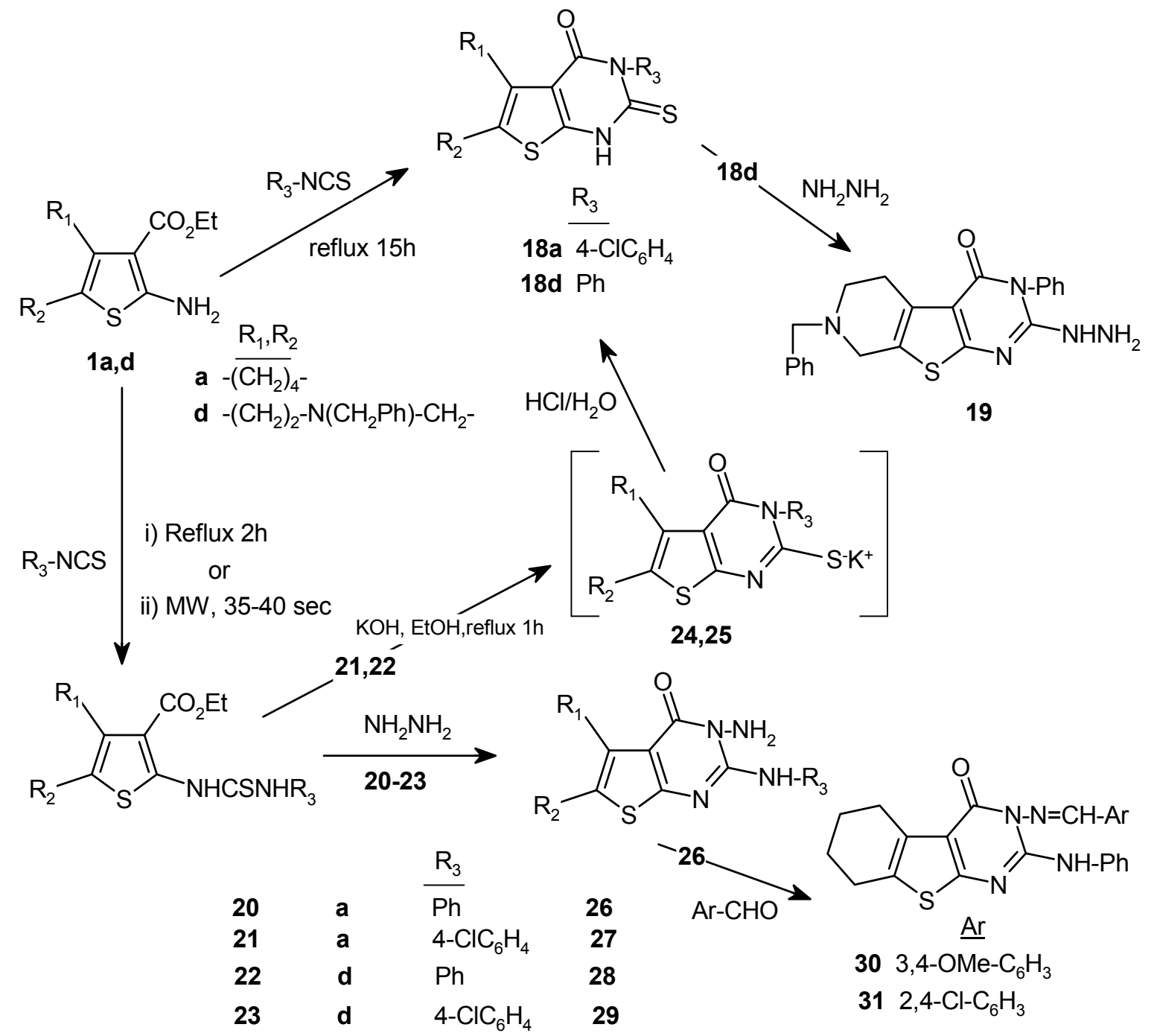

The thioureido derivatives $\mathbf{2 0 - 2 3}$ were obtained in moderate yields upon reaction of $\mathbf{1 a , d}$ with some aromatic isothiocyanates using either the classical heating method [12] or microwave irradiation [13], although the reaction was cleaner in the case of the latter method (Scheme 3). Thioureido compounds $\mathbf{2 1}$ and $\mathbf{2 2}$ were transformed to thienopyrimidine $\mathbf{1 8}$ through the corresponding pyridinium salts $\mathbf{2 4}$ and 25, respectively. Compounds 18a,d were obtained directly, although in low yields, on refluxing 1a,d with the aromatic isothiocyanates for longer times. Condensation of $\mathbf{1 8 d}$ with hydrazine hydrate gave the novel 2-hydrazino derivative 19. Compounds 20-23 were also condensed with hydrazine hydrate to give the corresponding 3-aminothienopyrimidines 26-29. The latter amines reacted smoothly with aromatic aldehydes, as exemplified by the reaction of $\mathbf{2 6}$ with two different aldehydes. Thienopyrimidines 28-31 have not been previously synthesized and their structures were firmly established on the basis of their NMR $\left(\delta_{\mathrm{H}, \mathrm{C}}\right)$ and mass spectral data (see Experimental).

The starting 2-amino-3-cyanothiophene derivatives 32a,b reacted with formamide to give the corresponding thienopyrimidine derivatives 33a,b (Scheme 4). Structural elucidation of the latter was based on the various spectroscopic methods and comparison of the NMR data of $\mathbf{3 3 b}$ with those given in the literature [13] for the same compound. 
Scheme 4. Synthesis of compounds 32-46.

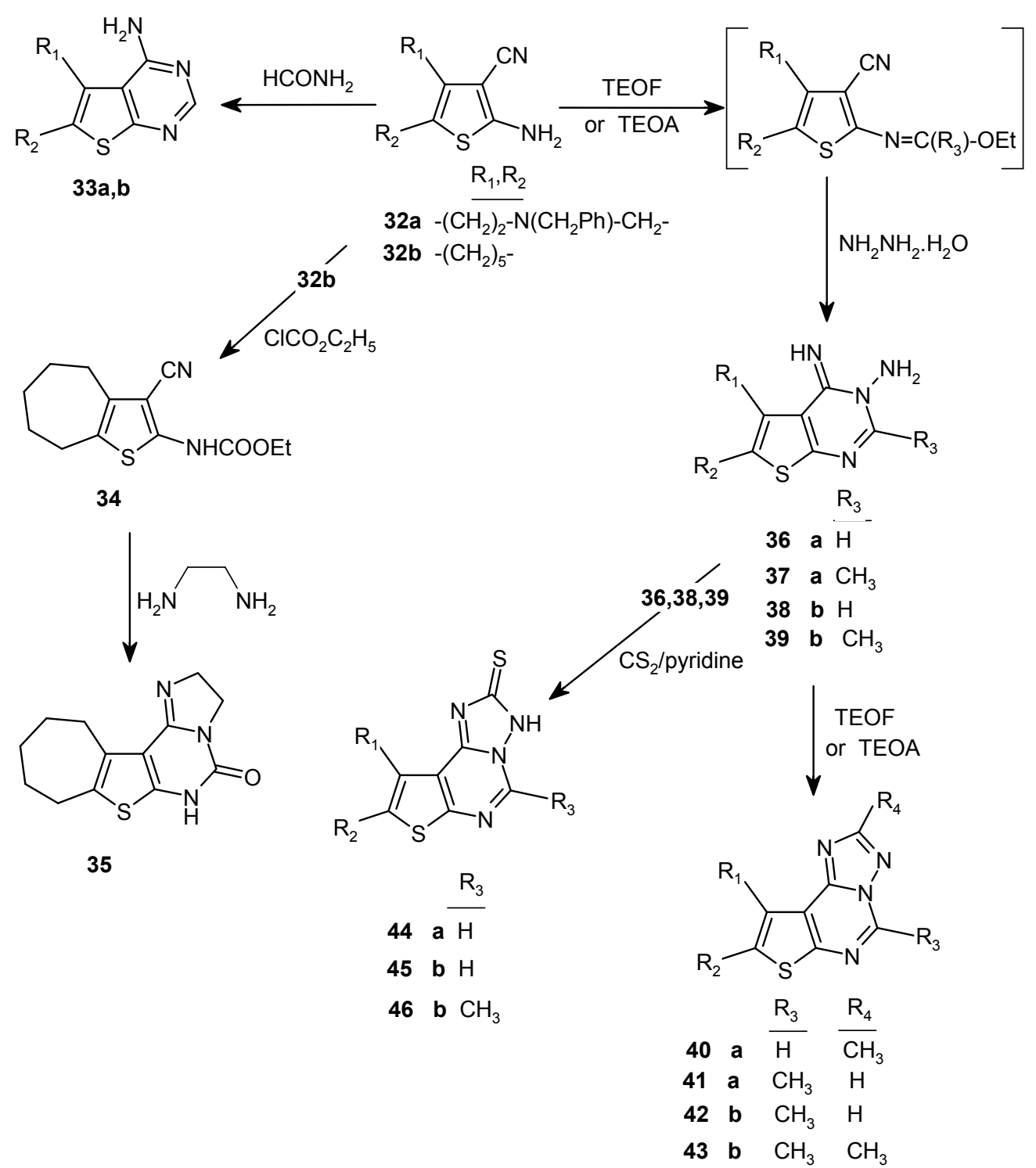

Further, condensation of 32a,b with TEOF and TEOA followed by hydrazine hydrate led to the formation of the novel thienopyrimidines 36-39, whose structures were elucidated from their IR, NMR and MS spectral data (see Experimental). Compounds 40-43 were obtained in moderate yields upon the reaction of 37-39 with TEOF and TEOA. Reaction of 36, 38 and 39 with carbon disulphide in pyridine gave the condensed thienopyrimidines 44-46. The structures of 42-46 were confirmed from their ${ }^{1} \mathrm{H}-\mathrm{NMR},{ }^{13} \mathrm{C}-\mathrm{NMR}$ and mass spectral data. The assignment of the NMR chemical shifts of the thienopyrimidines were made on the basis of COSY ${ }^{1} \mathrm{H}-\mathrm{NMR}$ and DEPT-135 experiments. HETCOR and HMBC spectra were further used in the structural elucidation of 38, 43, 45 and 46 . Thus, the ${ }^{1} \mathrm{H}-$ NMR spectrum of $\mathbf{4 3}$ displayed four multiplets and two singlets in the aliphatic region attributable to the protons in five methylenes and two methyl groups. The signals of these aliphatic carbons in the ${ }^{13} \mathrm{C}-\mathrm{NMR}$ spectrum were verified through the corresponding DEPT-135 technique. The remaining signals in the latter spectrum correspond to the aromatic carbons in $\mathbf{4 3}$, which were unambiguously assigned on the basis of the analysis of HETCOR and HMBC spectral data. Figure 3 shows the HMBC spectrum correlations in $\mathbf{4 3}$. 
Figure 3. Selected HMBC spectrum of 43.

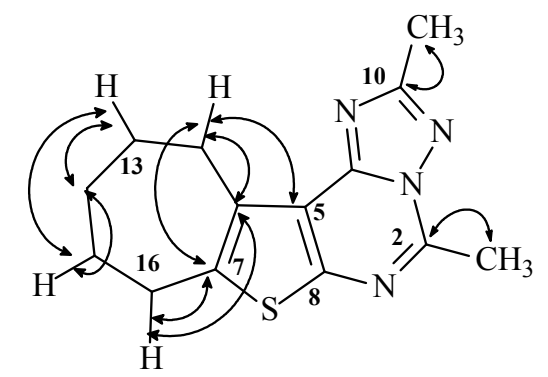

\subsection{Biological studies}

\subsubsection{Antibacterial screening}

Representative microbes [Staphylococcus aureus (ATCC 25923), Klepsela monas(ATCC 700603), Pseudomonas aeruginosa (ATCC 27584) and Escherichia coli (ATCC 25922)] were used as test organisms. The filter paper disc method $[16,17]$ was used for the antimicrobial screening of selected compounds (4a, 8c, 12, 15, 17, 41 and 46). The tested compounds were dissolved in a suitable solvent (the concentration of the compounds was 10\%). Standard blank paper discs (5 $\mathrm{mm}$ in diameter) were separately soaked in the solutions of each compound and after 3-5 min transferred onto the surface of growth media seeded with the test organism. After an incubation period under suitable conditions for the test organism $\left(35^{\circ} \mathrm{C}\right)$ and after $24 \mathrm{~h}$, the diameter of the inhibition zones around the discs were measured in millimeters. The effects were compared with the reference antibiotics vancomycin (VA) in the case of $S$. aureus and cefatzidine (CAZ) in the case of other tested organisms. The antibiotics VA and CAZ were used at a concentration of $20 \mathrm{mg} / \mathrm{mL}$ as references. The obtained results are summarized in Table 2. Most of the compounds (except 17) showed very good activity against Staphylococcus aureus comparing with the used reference standards. Some of the tested compounds showed very weak or moderate activities aginst Klepsela monas, Pseudomonas aeruginosa and Escherichia coli, as can be noticed from Table 2.

Table 2. Antimicrobial activity of the select synthesized compounds.

\begin{tabular}{|c|c|c|c|c|}
\hline & \multicolumn{4}{|c|}{ Diameter of inhibition zone (mm) } \\
\hline & \multicolumn{2}{|c|}{ Gram-positive } & K. $\boldsymbol{P}$ & Gram -negative \\
\hline $\mathbf{4 a}^{* * *}$ & 12 & 4 & 6 & E.c \\
\hline $\mathbf{8 c}^{* * *}$ & 16 & 0 & 0 & 13 \\
\hline $\mathbf{1 2}^{*}$ & 12 & 4 & 0 & 4 \\
\hline $\mathbf{1 5}^{*}$ & 11 & 7 & 4 & 6 \\
\hline $\mathbf{1 7}^{*}$ & 0 & 0 & 4 & 0 \\
\hline $\mathbf{4 1}^{*}$ & 9 & 2 & 1 & 0 \\
\hline $\mathbf{4 6}^{* *}$ & 9 & 3 & 7 & 2 \\
\hline $\mathbf{C A Z}$ & - & 9 & 28 & 20 \\
\hline VA & 17 & - & - & - \\
\hline
\end{tabular}

$* \mathrm{DMF} ; * \mathrm{CHCl}_{3}$. 


\subsubsection{Antitumor studies}

The synthesized thienopyrimidines $4 \mathbf{a}, \mathbf{7 d}, \mathbf{8 a}, \mathbf{1 2}, \mathbf{1 5}, \mathbf{2 9}$ and $\mathbf{4 2}$ were tested against the following human tumor cell lines: colorectal carcinoma (HCT116), hepatocellular carcinoma (HEPG2), cervix adenocarcinoma (HELA), larynx carcinoma (HEP2), human breast adenocarcinoma (MCF7). The drug doxorubicin [18] has been used as a reference in the present study to compare the inhibition effect for tested compounds on the growing cancer cells. Measurement of Potential Cytotoxicity by the SRB Assay was used [19]. Compounds 8, 12 and 29 showed very good anticancer activity against HEPG2, while most of the compounds were very active against HELA, except $\mathbf{4 a}$ and $7 \mathrm{~d}$. Compounds $\mathbf{7 d}$ and $\mathbf{1 5}$ were active toward MCF7. For cell line HCT116 only 7d and $\mathbf{4 2}$ were very active, on the other hand 7d, 8a and 15 were very active against HEP2. Results are summarized in Table 3.

Table 3. Results of antitumor cytotoxicity for some synthesized thienopyrimidines.

\begin{tabular}{|c|c|c|c|c|c|}
\hline \multirow{2}{*}{ Comp. No } & \multicolumn{5}{|c|}{ Cytotoxicity IC $_{\mathbf{5 0}}(\mathbf{\mu g} / \mathbf{m L})$} \\
\cline { 2 - 6 } & HEPG2 & HELA & MCF7 & HCT116 & HEP2 \\
\hline $\mathbf{4 a}$ & 2.35 & 1.07 & 2.82 & 2.01 & 1.61 \\
$\mathbf{7 d}$ & 1.28 & 1.41 & 0.67 & 0.47 & 0.47 \\
$\mathbf{8 a}$ & 0.94 & 0.87 & 0.74 & - & 0.47 \\
$\mathbf{1 2}$ & 0.74 & 0.81 & 1.54 & 2.68 & 0.81 \\
$\mathbf{1 5}$ & 1.21 & 0.40 & 0.6 & 1.68 & 0.54 \\
$\mathbf{2 9}$ & 0.40 & 0.60 & 0.94 & 2.89 & 1.14 \\
$\mathbf{4 2}$ & 1.81 & 0.81 & 2.48 & 0.54 & 1.14 \\
Doxorubicin & 0.54 & 0.85 & 0.7 & 0.69 & 0.4 \\
\hline
\end{tabular}

\section{Experimental}

\subsection{General}

Melting points were determined on an Electrothermal IA9000 series digital capillary melting point apparatus. IR spectra were run $\left(\mathrm{KBr}\right.$ discs) on a Shimadzu FT spectrophotometer $1000 .{ }^{1} \mathrm{H}$ - and ${ }^{13} \mathrm{C}$ NMR spectra were recorded in $\mathrm{DMSO}_{-} \mathrm{d}_{6}\left(\right.$ or in $\mathrm{CDCl}_{3}$ ) on a JEOL ECP $400 \mathrm{NMR}$ spectrometer operating at $400 / 100 \mathrm{MHz}$, with TMS as internal standard. DEPT and HETCOR experiments were recorded on a $500 \mathrm{MHz}$ instrument (Bruker, J.F.B. 288) at King Saud University (Pharmacy Research Centre). Chemical shifts are given in $\delta(\mathrm{ppm})$ and coupling constants $(J)$ are given in Hz. The assignments of all carbons are made by comparison to ${ }^{13} \mathrm{C}-\mathrm{NMR}$ spectra of structurally related compounds [12,13] and theoretical grounds [20,21], and with the aid various modern NMR techniques in many cases. Electron impact (EI) MS spectra were recorded on a Shimadzu GCMSQP5050A spectrometer (DB-1 glass column $30 \mathrm{~m} \times 0.25 \mathrm{~mm}$, ionization energy $70 \mathrm{eV}$ ), at the Chemistry Department, College of Science, King Saud University. Antimicrobial and anti-cancer tests of some of the synthesized compounds were run in King Abdul Aziz Hospital for the National Guard, Al-hasa, Saudi Arabia and the Pharmacology Unit, National Cancer Institute, Cairo University, Egypt, respectively. The reactions were monitored by TLC, and the purity of the compounds were routinely checked by TLC silica gel plates while the spots were visualised by UV (Uvitec). The starting 
materials, ethyl 2-amino-4,5-disubstituted thiophene-3-carboxylates 1a-d and the 2-amino-4,5disubstituted thiophene-3-carbonitrile 32, were prepared by condensation of ketones, elemental sulfur, ethyl cyanoacetate or malononitrile as described [14,15].

\subsection{General procedure for synthesis of $\mathbf{2 a , b}$}

Compounds 2a,b were both prepared according to a method reported in the literature [22] for similar compounds. 5-Chlorovaleryl chloride $(1.3 \mathrm{~mL}, 10 \mathrm{mmol})$ was added to a solution of amino ester 1a,b $(10 \mathrm{mmol})$ in chloroform $(20 \mathrm{~mL})$ and the solution was refluxed for $4 \mathrm{~h}$. After cooling, the solution was concentrated under reduced pressure to give a dark oil. Addition of a small amount of water and ethanol yielded a solid that was collected, dried and recrystallized from ethanol.

2-(5-Chloropentanoylamino)-4,5,6,7-tetrahydrobenzo[b]-thiophene-3-carboxylic acid ethyl ester (2a). Colorless crystals, m.p. 54-55 ${ }^{\circ} \mathrm{C}$; yield 86\%; IR $\left(\mathrm{cm}^{-1}\right): 3,230(\mathrm{NH}), 1,678(\mathrm{CO}$, ester), 1,662 (CO, amide); ${ }^{1} \mathrm{H}-\mathrm{NMR}\left(\mathrm{CDCl}_{3}\right) \delta: 1.36\left(3 \mathrm{H}, \mathrm{t}, J=7.3 \mathrm{~Hz}, \mathrm{CH}_{2} \mathrm{CH}_{3}\right), 1.76\left(4 \mathrm{H}, \mathrm{m}, 2 \mathrm{CH}_{2}\right), 1.86(4 \mathrm{H}, \mathrm{m}$, $\left.2 \mathrm{CH}_{2}\right), 2.48\left(2 \mathrm{H}, \mathrm{t}, J=7.0 \mathrm{~Hz}, \mathrm{CH}_{2}-\mathrm{CO}\right), 2.61\left(2 \mathrm{H}, \mathrm{t}, J=5.5 \mathrm{~Hz}, \mathrm{CH}_{2}\right), 2.74\left(2 \mathrm{H}, \mathrm{t}, J=5.5 \mathrm{~Hz}, \mathrm{CH}_{2}\right)$, $3.54\left(2 \mathrm{H}, \mathrm{t}, J=7 \mathrm{~Hz}, \mathrm{CH}_{2} \mathrm{Cl}\right), 4.29\left(2 \mathrm{H}, \mathrm{q}, J=7.3 \mathrm{~Hz}, \mathrm{CH}_{2} \mathrm{CH}_{3}\right), 11.01(\mathrm{~s}, \mathrm{NH}) ;{ }^{13} \mathrm{C}-\mathrm{NMR}\left(\mathrm{CDCl}_{3}\right) \delta$ : 22.61, 26.92, 26.44, $31.89\left(4 \mathrm{C}, 4 \mathrm{CH}_{2}\right), 23.05,24.71,35.90,44.47\left(4 \mathrm{C}, \mathrm{CH}_{2}\right) 14.38\left(\mathrm{CH}_{2} \mathrm{CH}_{3}\right), 60.54$ $\left(\mathrm{CH}_{2} \mathrm{CH}_{3}\right), 111.46,126.75,130.78,147.54$ (4C, thiophene carbons), 166.75 (CO-NH), 169.32 (ester $\mathrm{CO})$.

2-(5-Chloropentanamido)-5,6,7,8-tetrahydro-4H-cyclohepta[b] thiophene-3-carboxylic acid ethyl ester (2b). Colorless crystals, m.p. $50{ }^{\circ} \mathrm{C}$; yield 86\%; IR $\left(\mathrm{cm}^{-1}\right): 3,363(\mathrm{NH}), 1,697$ (CO, ester), 1,681 (CO, amide); ${ }^{1} \mathrm{H}-\mathrm{NMR}\left(\mathrm{CDCl}_{3}\right) \delta: 1.35\left(3 \mathrm{H}, \mathrm{t}, J=7 \mathrm{~Hz}, \mathrm{CH}_{2} \mathrm{CH}_{3}\right), 1.60\left(4 \mathrm{H}, \mathrm{m}, 2 \mathrm{CH}_{2}\right), 1.83(4 \mathrm{H}, \mathrm{m}$, $\left.2 \mathrm{CH}_{2}\right), 2.45\left(2 \mathrm{H}, \mathrm{t}, J=6.9 \mathrm{~Hz}, \mathrm{CH}_{2}-\mathrm{CO}\right), 2.67\left(2 \mathrm{H}, \mathrm{m}, \mathrm{CH}_{2}\right), 2.99\left(2 \mathrm{H}, \mathrm{m}, \mathrm{CH}_{2}\right), 3.52(2 \mathrm{H}, \mathrm{t}$, $\left.J=6.9 \mathrm{~Hz}, \mathrm{CH}_{2} \mathrm{Cl}\right), 4.29\left(2 \mathrm{H}, \mathrm{q}, J=7 \mathrm{~Hz}, \mathrm{CH}_{2} \mathrm{CH}_{3}\right), 11.18(1 \mathrm{H}, \mathrm{s}, \mathrm{NH}) ;{ }^{13} \mathrm{C}-\mathrm{NMR}\left(\mathrm{CDCl}_{3}\right) \delta: 22.63$, 26.99, 27.86, 31.87, $32.24\left(5 \mathrm{C}, 5 \mathrm{CH}_{2}\right), 28.31,28.62,35.89,44.46\left(4 \mathrm{C}, \mathrm{CH}_{2}\right) 14.38\left(\mathrm{CH}_{2} \mathrm{CH}_{3}\right), 60.72$ $\left(\mathrm{CH}_{2} \mathrm{CH}_{3}\right), 112.82,130.95,136.36,145.45$ (4C, thiophene carbons), $169.29(\mathrm{CO}), 166.78(\mathrm{CO}-\mathrm{NH})$.

\subsection{2-[5-(4-(2-Methoxyphenyl)-1-piperazin-1-yl]pentanoylamino)-4,5,6,7-tetrahydrobenzo[b]-thio- phene-3-carboxylic acid ethyl ester (3a)}

A mixture of 2a (3.9 mmol), 1-(2-methoxyphenyl)piperazine (750 mg, $3.9 \mathrm{mmol})$, and potassium carbonate $(540 \mathrm{mg}, 3.9 \mathrm{mmol})$ was dissolved in DMF $(7 \mathrm{~mL})$ and refluxed under stirring for $2 \mathrm{~h}$. After cooling, the suspension was filtered and the solution was extracted with chloroform and washed with water. The organic layers were dried over anhydrous sodium sulfate and evaporated under reduced pressure. Recrystallization from ethanol gave white crystals, m.p. $105-106{ }^{\circ} \mathrm{C}$; yield $94 \%$; IR $\left(\mathrm{cm}^{-1}\right)$ : 3,261 (NH), 1,691 (CO, ester), 1,660 (CO, amide); ${ }^{1} \mathrm{H}-\mathrm{NMR}\left(\mathrm{CDCl}_{3}\right) \delta: 1.34(3 \mathrm{H}, \mathrm{t}, J=7.32 \mathrm{~Hz}$, $\left.\mathrm{CH}_{2} \mathrm{CH}_{3}\right), 1.60\left(2 \mathrm{H}, \mathrm{m}, \mathrm{CH}_{2}\right), 1.75\left(6 \mathrm{H}, \mathrm{m}, 3 \mathrm{CH}_{2}\right), 2.43\left(4 \mathrm{H}, \mathrm{t}, J=7.3 \mathrm{~Hz}, 2 \mathrm{CH}_{2}-\mathrm{N}\right.$ piperazine), 2.48 (4H, t, $J=7.3 \mathrm{~Hz}, 2 \mathrm{CH}_{2}-\mathrm{N}$ piperazine), $2.61\left(6 \mathrm{H}, \mathrm{br}, 3 \mathrm{CH}_{2}\right), 2.73\left(2 \mathrm{H}, \mathrm{br} \mathrm{s}, \mathrm{CH}_{2}-\mathrm{CO}\right), 3.81(3 \mathrm{H}, \mathrm{s}$, $\left.\mathrm{O}-\mathrm{CH}_{3}\right), 4.28\left(2 \mathrm{H}, \mathrm{q}, J=7.3 \mathrm{~Hz}, \mathrm{CH}_{2} \mathrm{CH}_{3}\right), 6.80-6.97(4 \mathrm{H}, \mathrm{m}, \mathrm{Ar}-\mathrm{H}), 11.01(\mathrm{~s}, \mathrm{NH}) ;{ }^{13} \mathrm{C}-\mathrm{NMR}$ $\left(\mathrm{CDCl}_{3}\right) \delta:$ 22.95, 23.07, 23.40, $24.42\left(4 \mathrm{C}, 4 \mathrm{CH}_{2} \mathrm{~N}\right.$-piperazine), 26.46, 36.80, 50.72, 53.55 (4C, 4 $\left.\mathrm{CH}_{2}\right), 55.40\left(\mathrm{OCH}_{3}\right), 26.46(2 \mathrm{C}), 58.26(2 \mathrm{C})\left(4 \mathrm{C}, 4 \mathrm{CH}_{2}\right), 14.41\left(\mathrm{CH}_{2} \mathrm{CH}_{3}\right), 60.50\left(\mathrm{CH}_{2} \mathrm{CH}_{3}\right), 111.30$, 
126.60, 130.71, 147.74 (4 C, thiophene carbons), 111.19, 118.22, 121.02, 122.88, 141.46, 152.32 (phenyl carbons), $166.75(\mathrm{CO}-\mathrm{NH}), 169.32(\mathrm{CO})$.

\subsection{2-(5-(4-(2-Methoxyphenyl)piperazin-1-yl)pentanamido)-5,6, 7,8-tetrahydro-4H-cyclohepta[b]-} thiophene-3-carboxylic acid ethyl ester (3b)

Compound $\mathbf{3 b}$ was prepared from equimolar amounts of $\mathbf{2 b}$ and 1-(2-methoxyphenyl)piperazine following the same conditions and work up described for the preparation of 3a. The product could not be induced to crystallize, although the NMR spectra proved the structure. Yield $85 \%$; ${ }^{1} \mathrm{H}-\mathrm{NMR}$ $\left(\mathrm{CDCl}_{3}\right) \delta: 1.72\left(4 \mathrm{H}, \mathrm{m}, 2 \mathrm{CH}_{2}\right), 1.84\left(2 \mathrm{H}, \mathrm{m}, \mathrm{CH}_{2}\right), 2.55\left(2 \mathrm{H}, \mathrm{m}, \mathrm{CH}_{2}\right), 2.85\left(2 \mathrm{H}, \mathrm{m}, \mathrm{CH}_{2}\right) ;{ }^{13} \mathrm{C}-\mathrm{NMR}$ $\left(\mathrm{CDCl}_{3}\right) \delta: 19.64,26.20,28.62,29.15$ (2C) (cycloheptane carbons).

\subsection{3-Amino-2-[4-[4-(2-methoxyphenyl)-piperazin-1-yl]butyl]-5,6,7,8-tetrahydro-3H-benzo[4,5]- thieno[2,3-d]pyrimidin-4-one (4a)}

A mixture of 3a $(20 \mathrm{mmol})$ and hydrazine hydrate $(4 \mathrm{~mL}, 80 \mathrm{mmol})$ was refluxed for $12 \mathrm{~h}$ in ethanol $(10 \mathrm{~mL})$. After cooling, the product was collected and washed with ethanol, dried and recrystallized from ethanol, to yield 4 a colorless crystals, m.p. $163-164{ }^{\circ} \mathrm{C}$; yield $92 \%$; IR $\left(\mathrm{cm}^{-1}\right)$ : 3,273 and 3,145 (NH), 1,672 (C=O); ${ }^{1} \mathrm{H}$ and ${ }^{13} \mathrm{C}$-NMR data: see Table 1; MS: $m / z 467\left(\mathrm{M}^{+}, 2.02 \%\right)$, $\left.\left.452\left(\mathrm{M}^{+}-\mathrm{CH}_{3}\right), 18.27 \%\right), 204\left(\mathrm{M}^{+}-\left(\mathrm{NH}_{2},\left(\mathrm{CH}_{2}\right)_{4}-\mathrm{N}_{2} \mathrm{C}_{4} \mathrm{H}_{8}-\mathrm{C}_{6} \mathrm{H}_{4}-\mathrm{OCH}_{3}\right)\right), 24.29 \%\right), 119\left(\mathrm{M}^{+}=\left[\mathrm{C}_{6} \mathrm{H}_{4}-\mathrm{N}-\right.\right.$ $\left.\left.\mathrm{CH}_{2} \mathrm{CH}_{3}\right]^{+}, 100 \%\right)$.

\subsection{3-Amino-2-[4-[4-(2-methoxyphenyl)-piperazin-1-yl]butyl]-6,7,8,9-tetrahydro-3H,5H- [4,5] thieno[2,3-d]pyrimidin-4-one (4b)}

This compound was prepared from $\mathbf{3 b}$ following the same conditions and work up described for the preparation of 4a. Colorless crystals, m.p. $183-185{ }^{\circ} \mathrm{C}$; yield $81 \%$; IR $\left(\mathrm{cm}^{-1}\right)$ : $3310,3135(\mathrm{NH}), 1670$ $(\mathrm{C}=\mathrm{O}) ;{ }^{1} \mathrm{H}$ and ${ }^{13} \mathrm{C}$-NMR data: almost the same data as $4 \mathbf{a}$ above except for an additional $\mathrm{CH}_{2}$ in the cycloheptane moiety in $\mathbf{4 b}: \delta_{\mathrm{H}} 1.79\left(4 \mathrm{H}, \mathrm{m}, 2 \mathrm{CH}_{2}\right), 1.95\left(2 \mathrm{H}, \mathrm{m}, \mathrm{CH}_{2}\right), 2.51\left(2 \mathrm{H}, \mathrm{m}, \mathrm{CH}_{2}\right), 2.87(2 \mathrm{H}$, $\left.\mathrm{m}, \mathrm{CH}_{2}\right)$ and $\delta_{\mathrm{C}} 19.81,25.14,27.40,29.15$ (2C, cycloheptane carbons); MS: $m / z 481\left(\mathrm{M}^{+}, 7.3 \%\right), 466$ $\left.\left(\mathrm{M}^{+}-\mathrm{CH}_{3}\right), 15.39 \%\right)$.

\subsection{2-(3'-Ethoxycarbonyluriedo)-3-ethoxycarbonyl-5,6,7,8-tetrahydro-4H-cyclohepta[b] thiophene (5)}

A mixture of $1 \mathbf{b}$ (1.195 g, $5 \mathrm{mmol})$ and ethyl isocyanatoformate $(575 \mathrm{mg}, 5 \mathrm{mmol})$ was placed in a $50 \mathrm{~mL}$ conical flask covered with a funnel glass and then irradiated with microwaves $(950 \mathrm{~W})$ for 35 seconds. The cold reaction mixture was treated with ethanol and the solid product was filtered and recrystallized from ethanol-water to give colorless needles, m.p. $167-169{ }^{\circ} \mathrm{C}$; yield 88\%; IR $\left(\mathrm{cm}^{-1}\right): 3,207,3,115(2 \mathrm{NH}), 1,739$ (CO, ester), 1,678, 1,675 (2 CO, amide); ${ }^{1} \mathrm{H}-\mathrm{NMR}\left(\mathrm{CDCl}_{3}\right) \delta: 1.32$ $\left(3 \mathrm{H}, \mathrm{t}, \mathrm{CH}_{3}\right), 1.36\left(3 \mathrm{H}, \mathrm{t}, \mathrm{CH}_{3}\right), 1.64\left(4 \mathrm{H}, \mathrm{m}, 2 \mathrm{CH}_{2}\right), 1.83\left(2 \mathrm{H}, \mathrm{m}, \mathrm{CH}_{2}\right), 2.71\left(2 \mathrm{H}, \mathrm{t}, \mathrm{CH}_{2}\right), 3.05(2 \mathrm{H}, \mathrm{t}$, $\left.\mathrm{CH}_{2}\right), 4.30$ and 4.40 (each $\left.2 \mathrm{H}, \mathrm{q}, \mathrm{O}-\mathrm{CH}_{2}\right), 7.45(1 \mathrm{H}, \mathrm{s}, \mathrm{NH}), 12.43(1 \mathrm{H}, \mathrm{s}, \mathrm{NH}) ;{ }^{13} \mathrm{C}-\mathrm{NMR}\left(\mathrm{CDCl}_{3}\right) \delta$ : 14.34 and 14.44 (each $\left.3 \mathrm{H}, \mathrm{t}, \mathrm{CH}_{3}\right), 27.06,27.89,28.35,28.69,32.33\left(5 \mathrm{C}-5 \mathrm{CH}_{2}\right), 60.72,62.98(2 \mathrm{C}$, O$\mathrm{CH}_{2}$ ), 114.22, 131.20, 137.18, 144.16 (thiophene ring), 165.52 (CO), 153.11, 149.56 (2 CO-amide); 
MS: $m / z 354\left(\mathrm{M}^{+}, 89.5 \%\right), 265\left(\mathrm{M}^{+}-\mathrm{NHCO}_{2} \mathrm{C}_{2} \mathrm{H}_{5}, \mathrm{H}, 23 \%\right), 192\left(\mathrm{M}^{+}-\mathrm{NHCO}_{2} \mathrm{C}_{2} \mathrm{H}_{5}, \mathrm{H}, \mathrm{CO}_{2} \mathrm{C}_{2} \mathrm{H}_{5}\right.$, $100 \%), 164\left(\mathrm{M}^{+}-\mathrm{NHCO}_{2} \mathrm{C}_{2} \mathrm{H}_{5}, \mathrm{H}, \mathrm{CO}_{2} \mathrm{C}_{2} \mathrm{H}_{5}, \mathrm{CO}, 74 \%\right)$.

\subsection{1,5,6, 7,8-Hexahydro-3H-cyclohepta[4,5]thienopyrimidin-2,4-dione (6)}

Compound 5 (1770 mg, $5 \mathrm{mmol}$ ) was added to a solution of sodium ethoxide [sodium metal $(120 \mathrm{mg})$, absolute ethanol $(15 \mathrm{~mL})]$, and refluxed $0.5 \mathrm{~h}$, then the solvent was removed under reduced pressure. The residue was treated with water, then acidified with dil. $\mathrm{HCl} \mathrm{1:1}(\mathrm{pH}=4)$, and the solid formed was collected and recrystallized from ethanol to give $\mathbf{6}$ as colorless crystals, m.p. $>300{ }^{\circ} \mathrm{C}$; yield 82\%; IR $\left(\mathrm{cm}^{-1}\right): 3,095,3,153(2 \mathrm{NH}), 1,708,1,666$ (2 $\left.\mathrm{CO}\right), 2,600-3,300(\mathrm{OH})$; ${ }^{1} \mathrm{H}-\mathrm{NMR}$ $\left(\mathrm{DMSO}_{6}\right) \delta: 1.53\left(4 \mathrm{H}, \mathrm{m}, 2 \mathrm{CH}_{2}\right), 1.80\left(2 \mathrm{H}, \mathrm{m}, \mathrm{CH}_{2}\right), 2.69\left(2 \mathrm{H}, \mathrm{t}, \mathrm{CH}_{2}\right), 3.10\left(2 \mathrm{H}, \mathrm{t}, \mathrm{CH}_{2}\right), 10.95(\mathrm{~s}$, $\mathrm{NH}), 11.75(\mathrm{NH}) ;{ }^{13} \mathrm{C}-\mathrm{NMR}\left(\mathrm{DMSO}_{6}\right) \delta: 27.21,27.31,27.98,28.87,32.41\left(5 \mathrm{C}, 5 \mathrm{CH}_{2}\right), 113.92$, 129.56, 136.79, 149.83 (thiophene carbons), 150.87, 160.73 (2 CO); MS: $m / z 236\left(\mathrm{M}^{+}, 100 \%\right), 208$ $\left.\left.\left(\mathrm{M}^{+}-\mathrm{C}_{2} \mathrm{H}_{4}, 39.4 \%\right), 193\left(\mathrm{M}^{+}-\mathrm{C}_{2} \mathrm{H}_{4}, \mathrm{NH}\right), 36 \%\right), 165\left(\mathrm{M}^{+}-\mathrm{C}_{2} \mathrm{H}_{4}, \mathrm{NH}, \mathrm{CO}\right), 48 \%\right), 137\left(\mathrm{M}^{+}-\mathrm{C}_{2} \mathrm{H}_{4}, \mathrm{NH}\right.$, $2 \mathrm{CO}), 42.4 \%), 133\left(\mathrm{M}^{+}-\left(\mathrm{C}_{2} \mathrm{H}_{4}, \mathrm{NH}, 2 \mathrm{CO}, \mathrm{H}\right), 33.3 \%\right)$.

\subsection{General procedure for synthesis of 5,6-disubstituted-3H-thieno[2,3-d] pyrimidin-4-ones 7a-d}

A mixture of 1a-d $(2 \mathrm{mmol})$ and formamide $(20 \mathrm{~mL})$ was heated under reflux for $1.5 \mathrm{~h}$, then left to cool to room temperature overnight. The solid formed was filtered, washed with water, dried and recrystallized from ethanol [13].

5,6,7,8-Tetrahydro-3H-benzo[4,5]thieno[2,3-d]pyrimidin-4-one (7a). Fine brown needles, m.p. 224-226 ${ }^{\circ} \mathrm{C}$; yield 92\%; IR $\left(\mathrm{cm}^{-1}\right)$ : 3,157 (NH), 3,007 (C-H), 1,658 (CO), 1,589 (C=C); ${ }^{1} \mathrm{H}-\mathrm{NMR}$ $\left(\mathrm{DMSO}_{6} \mathrm{~d}_{6}\right) \delta: 1.76\left(4 \mathrm{H}, \mathrm{m}, 2 \mathrm{CH}_{2}\right), 2.71\left(2 \mathrm{H}, \mathrm{t}, \mathrm{CH}_{2}\right), 2.86\left(2 \mathrm{H}, \mathrm{t}, \mathrm{CH}_{2}\right), 7.98(\mathrm{H}-2), 12.29$ (br, s, NH); ${ }^{13} \mathrm{C}-\mathrm{NMR}\left(\mathrm{DMSO}_{6}\right)$ ) $: 22.31,23.00,24.98,25.88$ (aliphatic carbons), 123.23, 131.35, 132.64, $145.37\left(4 \mathrm{C}\right.$, thiophene carbons), $158.21(\mathrm{C}=\mathrm{N}), 162.96(\mathrm{C}=\mathrm{O})$; MS: $m / z 206\left(\mathrm{M}^{+}, 20 \%\right), 178\left(\mathrm{M}^{+}-\right.$ $(\mathrm{HCNH}), 1 \%), 57\left(\mathrm{C}_{2} \mathrm{SH}, 100 \%\right)$.

3,5,6,7,8,9-Hexahydrocyclohepta[4,5]thieno[2,3-d]pyrimidin-4-one (7b). Fine brown crystals, m.p. 118-220 ${ }^{\circ} \mathrm{C}$; yield 90\%; IR $\left(\mathrm{cm}^{-1}\right): 3,151(\mathrm{NH}), 3,012(\mathrm{C}-\mathrm{H}), 1,656(\mathrm{CO}), 1,598(\mathrm{C}=\mathrm{C}) ;{ }^{1} \mathrm{H}-\mathrm{NMR}$ $\left(\mathrm{DMSO}_{6}\right) \delta: 1.60\left(4 \mathrm{H}, \mathrm{m}, 2 \mathrm{CH}_{2}\right), 1.84\left(2 \mathrm{H}, \mathrm{m}, \mathrm{CH}_{2}\right), 2.82\left(2 \mathrm{H}, \mathrm{m}, \mathrm{CH}_{2}\right), 3.25\left(2 \mathrm{H}, \mathrm{t}, \mathrm{CH}_{2}\right), 7.98(\mathrm{H}-$ 2), 12.30 (br, s, NH); ${ }^{13} \mathrm{C}-\mathrm{NMR}\left(\mathrm{DMSO}_{\mathrm{d}}\right.$ ) $\delta: 27.70$ (2C), 27.81, 29.57, 32.50 (aliphatic carbons), 123.78, 136.98, 145.07, $149.00(4 \mathrm{C}$, thiophene carbons), $158.75(\mathrm{C}=\mathrm{N}), 161.40(\mathrm{C}=\mathrm{O}) ; \mathrm{MS}: m / z 220$ $\left(\mathrm{M}^{+}, 63 \%\right), 192\left(\mathrm{M}^{+}-\mathrm{C}_{2} \mathrm{H}_{4}, 45 \%\right), 165\left(\mathrm{M}^{+}-\mathrm{C}_{2} \mathrm{H}_{4}, \mathrm{HCN}, 39 \%\right), 122\left(\mathrm{M}^{+}-\mathrm{C}_{2} \mathrm{H}_{4}, \mathrm{HCN}, \mathrm{CO}, \mathrm{NH}, 27 \%\right), 58$, $\left.\mathrm{C}_{2} \mathrm{H}_{2} \mathrm{~S}, 100 \%\right)$.

5,6-Dimethylthieno[2,3-d]pyrimidin-4-one (7c). Fine yellow crystals, m.p. 269-270 ${ }^{\circ} \mathrm{C}$; yield 93\%; IR $\left(\mathrm{cm}^{-1}\right)$ : 3,151 (NH), 3,057 (C-H), 1,656 (CO), 1,558 (C=C); ${ }^{1} \mathrm{H}-\mathrm{NMR}\left(\mathrm{DMSO}_{\mathrm{d}}\right) \delta$ : $2.33(3 \mathrm{H}, \mathrm{s}$, $\left.\mathrm{CH}_{3}\right), 2.37\left(3 \mathrm{H}, \mathrm{s}, \mathrm{CH}_{3}\right), 7.98(1 \mathrm{H}, \mathrm{s}, \mathrm{CH}), 12.29$ (br, s, NH); ${ }^{13} \mathrm{C}-\mathrm{NMR}\left(\mathrm{DMSO}-\mathrm{d}_{6}\right) \delta: 13.09,13.40$ $\left(2 \mathrm{C}, \mathrm{CH}_{3}\right), 129.25,129.79,132.64,145.23(4 \mathrm{C}$, thiophene carbons), $158.49(\mathrm{C}=\mathrm{N}), 162.19(\mathrm{C}=\mathrm{O})$; MS: $m / z 180\left(\mathrm{M}^{+}, 100 \%\right), 165\left(\mathrm{M}^{+}-\mathrm{CH}_{3}, 1 \%\right), 57\left(\mathrm{C}_{2} \mathrm{SH}, 94 \%\right)$. 
7-Benzyl-5,6,7,8-tetrahydro-3H-pyrido[4,3 :4,5]thieno[2,3-d]pyrimidin-4-one (7d). Fine yellow crystals, m.p. $234-235{ }^{\circ} \mathrm{C}$; yield 89\%; IR $\left(\mathrm{cm}^{-1}\right): 3,157(\mathrm{NH}), 3,068(\mathrm{C}-\mathrm{H}), 1,660(\mathrm{CO}), 1,581(\mathrm{C}=\mathrm{C})$; ${ }^{1} \mathrm{H}-\mathrm{NMR}\left(\mathrm{DMSO}_{6}\right) \delta: 2.75\left(2 \mathrm{H}, \mathrm{t}, \mathrm{CH}_{2}\right), 2.93\left(2 \mathrm{H}, \mathrm{t}, \mathrm{CH}_{2}\right), 2.60\left(2 \mathrm{H}, \mathrm{s}, \mathrm{CH}_{2}\right), 2.69\left(2 \mathrm{H}, \mathrm{s}, \mathrm{CH}_{2}\right)$, 7.26-7.35 (5H, m, Ar-H), $8.02(1 \mathrm{H}, \mathrm{s}, \mathrm{CH}), 12.39$ (1H, br, s, NH); ${ }^{13} \mathrm{C}-\mathrm{NMR}$ : (DMSO-d 6 ) $\delta: ~ 26.20$, 49.58, 51.51, 61.38 (4C, aliphatic carbons), 127.65, 128.85 (2C), $129.34(2 \mathrm{C}), 129.80(\mathrm{Ar}-\mathrm{CH})$, 122.87, 130.38, 138.69, 145.74 (4C, thiophene carbons), $158.21(\mathrm{C}=\mathrm{N}) ; 163.43(\mathrm{C}=\mathrm{O}) ; \mathrm{Ms}: \mathrm{m} / z 297$ $\left(\mathrm{M}^{+}, 93 \%\right), 206\left(\mathrm{M}^{+}-\mathrm{CH}_{2}-\mathrm{C}_{6} \mathrm{H}_{5}, 32 \%\right), 178\left(\mathrm{M}^{+}-\mathrm{CH}_{2}-\mathrm{C}_{6} \mathrm{H}_{5}, \mathrm{HCN}, \mathrm{H}, 100 \%\right)$.

\subsection{General procedure for synthesis of 4,5-disubstituted-3H-thieno[2,3-d] pyrimidin-4-thiones 8a,c,d}

A mixture of compound 7a,c,d (10 mmol), phosphorous pentasulphide (4.02 g, $30 \mathrm{mmol})$ and dry pyridine $(50 \mathrm{~mL})$ was refluxed with stirring for $2 \mathrm{~h}$. The mixture was evaporated to dryness under reduced pressure and the residue was boiled with water $(100 \mathrm{~mL})$ for one hour. After cooling overnight in refrigerator, the formed solid was recrystallized from a suitable solvent.

5,6,7,8-Tetrahydro-3H-benzo[4,5]thieno[2,3-d]pyrimidin-4-thione (8a). Recrystallized from ethanol give yellow crystals, m.p. $235-237{ }^{\circ} \mathrm{C}$; yield 90\%; IR $\left(\mathrm{cm}^{-1}\right): 3,132(\mathrm{NH}), 3,061(\mathrm{Ar}-\mathrm{CH}), 1,562$ $(\mathrm{C}=\mathrm{C}), 1,185(\mathrm{C}=\mathrm{S}) ;{ }^{1} \mathrm{H}-\mathrm{NMR}\left(\mathrm{DMSO}_{-} \mathrm{d}_{6}\right) \delta: 1.61\left(4 \mathrm{H}, \mathrm{m}, 2 \mathrm{CH}_{2}\right), 2.53\left(2 \mathrm{H}, \mathrm{t}, \mathrm{CH}_{2}\right), 3.04(2 \mathrm{H}, \mathrm{t}$, $\mathrm{CH}_{2}$ ), $7.62(1 \mathrm{H}, \mathrm{s}, \mathrm{CH}), 13.14$ (br, s, NH); ${ }^{13} \mathrm{C}-\mathrm{NMR}\left(\mathrm{DMSO}_{6}\right)$ $\delta: 22.26,22.40,25,63,27.99$ (aliphatic carbons), 132.55, 132.91, 135.68, 142.42 (thiophene carbons); $161.18(\mathrm{C}=\mathrm{N}) ; 178.24(\mathrm{C}=\mathrm{S})$; MS: $m / z 222\left(\mathrm{M}^{+}, 100 \%\right), 180\left(\mathrm{M}^{+}-\mathrm{NCHNH}, 66 \%\right), 149\left(\mathrm{M}^{+}-\mathrm{NCHNH}, \mathrm{S},+\mathrm{H}, 66 \%\right)$.

5,6-Dimethyl-3H-thieno[2,3-d]pyrimidin-4-thione (8c). Light brown crystals, m.p. 247-248 ${ }^{\circ} \mathrm{C}$ (ethanol); yield 88\%; IR ( $\left.\mathrm{cm}^{-1}\right): 3,120(\mathrm{NH}), 3,068(\mathrm{Ar}-\mathrm{CH}), 1,568(\mathrm{C}=\mathrm{C}) 1,181(\mathrm{C}=\mathrm{S}) ;{ }^{1} \mathrm{H}-\mathrm{NMR}$ $\left(\mathrm{CDCl}_{3}\right) \delta: 2.32\left(3 \mathrm{H}, \mathrm{s}, \mathrm{CH}_{3}\right), 2.57\left(3 \mathrm{H}, \mathrm{s}, \mathrm{CH}_{3}\right), 8.09(1 \mathrm{H}, \mathrm{s}, \mathrm{CH}), 13.56(\mathrm{br}, \mathrm{s}, \mathrm{NH}) ;{ }^{13} \mathrm{C}-\mathrm{NMR}\left(\mathrm{CDCl}_{3}\right)$ $\delta$ : $13.05,15.05\left(2 \mathrm{C}, \mathrm{CH}_{3}\right), 130.29,131.97(2 \mathrm{C}), 143.13(4 \mathrm{C}$, thiophene carbons $), 160.13(\mathrm{C}=\mathrm{N})$, $178.09(\mathrm{C}=\mathrm{S})$; MS: $m / z 196\left(\mathrm{M}^{+}, 100 \%\right), 181\left(\mathrm{M}^{+}-\mathrm{CH}_{3}, 24 \%\right), 163\left(\mathrm{M}^{+}-\mathrm{SH}, 54 \%\right)$.

7-Benzyl-5,6,7,8-tetrahydro-3H-pyredo-[4;3 :4,5]thieno[2,3-d]pyrimidin-4-thione (8d). Yellow powder, m.p. 204-207 ${ }^{\circ} \mathrm{C}$ (ethanol-DMF); yield: 50\%; IR $\left(\mathrm{cm}^{-1}\right)$ : 3,415 (NH), 3,028 (Ar-CH), 1,180 (C=S), $1,629(\mathrm{C}=\mathrm{C}) ;{ }^{1} \mathrm{H}-\mathrm{NMR}\left(\mathrm{DMSO}_{\mathrm{d}}\right) \delta: 2.98\left(2 \mathrm{H}, \mathrm{CH}_{2}\right), 3.37\left(2 \mathrm{H}, \mathrm{CH}_{2}\right), 4.13\left(2 \mathrm{H}, \mathrm{CH}_{2}\right), 4.31(2 \mathrm{H}, \mathrm{s}$, $\mathrm{CH}_{2}-\mathrm{Ph}$ ), 7.23-7.39 (5H, m, Ar-H), 8,32 (H-2), 13.78 (br.s, NH).

\subsection{General procedure for the synthesis of $\mathbf{1 0 a}, \mathbf{c}, \mathbf{d}$}

A mixture of 1a,c,d $(10 \mathrm{mmol})$ and ethoxycarbonyl isothiocyanate $(10 \mathrm{mmol})$ in ethanol $(5 \mathrm{~mL})$ was placed in a $50 \mathrm{~mL}$ conical flask covered with a funnel glass and then irradiated with microwaves $(950 \mathrm{~W})$ for $35-40$ seconds. The cold reaction mixture was treated with ethanol and the solid product was filtered and recrystallized from a suitable solvent.

2-(Ethoxycarbonylamino-carbothioyl)amino-4,5,6,7-tetrahydrobenzo[4,5]thiophene-3-carboxylic acid ethyl ester (10a). Yellow crystals, m.p. $193-195{ }^{\circ} \mathrm{C}$ (ethanol); yield 92\%; IR (cm $\left.{ }^{-1}\right): 3,182(\mathrm{NH}), 1,732$ (ester CO), 1,680 (amide CO), 1,562, $1,533(\mathrm{C}=\mathrm{C}), 1,147(\mathrm{C}=\mathrm{S}), 1,201(\mathrm{C}-\mathrm{O}) ;{ }^{1} \mathrm{H}-\mathrm{NMR}\left(\mathrm{CDCl}_{3}\right) \delta$ : 
1.30 and 1.34 (each $3 \mathrm{H}, \mathrm{t}, J=7.3 \mathrm{~Hz}, \mathrm{CH}_{3} \mathrm{CH}_{2}$ ), 4.32 and 4.40 (each $2 \mathrm{H}, \mathrm{q}, J=7.3 \mathrm{~Hz}, \mathrm{CH}_{2} \mathrm{CH}_{3}$ ), 1.76 $\left(4 \mathrm{H}, \mathrm{m}, 2 \mathrm{CH}_{2}\right), 2.63\left(2 \mathrm{H}, \mathrm{t}, \mathrm{CH}_{2}\right), 2.77\left(2 \mathrm{H}, \mathrm{t}, \mathrm{CH}_{2}\right), 8.19$ (br. s, NH), $14.03(\mathrm{~s}, \mathrm{NH}) ;{ }^{13} \mathrm{C}-\mathrm{NMR}$ $\left(\mathrm{CDCl}_{3}\right)$ 8: 14.36, $14.44\left(2 \mathrm{C}, 2 \mathrm{CH}_{3}\right), 22,92\left(2 \mathrm{C}, 2 \mathrm{CH}_{2}\right), 24.45,26.49,60.81,63.09,116.09,128.31$, 131.78, 147.16 (thiophene carbons), $151.41(\mathrm{CO}-\mathrm{N}), 165.66(\mathrm{CO}), 173.27(\mathrm{C}=\mathrm{S})$.

2-(Ethoxycarbonylamino-carbothioyl)amino-4,5-dimethyl[4,5]thiophene-3-carboxylic acid ethyl ester (10c). Yellow crystals, m.p. $274-176{ }^{\circ} \mathrm{C}$ (ethanol); yield 90\%; IR $\left(\mathrm{cm}^{-1}\right)$ : 3,176 (NH), 1,728 $(\mathrm{C}=\mathrm{O})$, 1,683 (amide $\mathrm{C}=\mathrm{O}), 1,564,1,531(\mathrm{C}=\mathrm{C}), 1,170(\mathrm{C}=\mathrm{S}), 1,293(\mathrm{C}-\mathrm{O}) ;{ }^{1} \mathrm{H}-\mathrm{NMR}\left(\mathrm{CDCl}_{3}\right) \delta$ : 1.32 and 137 (each 3H, t, $\left.\mathrm{CH}_{3} \mathrm{CH}_{2}\right), 2.64\left(3 \mathrm{H}, \mathrm{s}, \mathrm{CH}_{3}-\mathrm{C} 4\right), 2.69\left(3 \mathrm{H}, \mathrm{s}, \mathrm{CH}_{3}-\mathrm{C} 5\right), 4.43\left(2 \mathrm{H}, \mathrm{q}, \mathrm{CH}_{2}-\mathrm{CH}_{3}\right), 4.32(2 \mathrm{H}$, q, $\left.\mathrm{CH}_{2}-\mathrm{CH}_{3}\right), 8.07(\mathrm{IH}, \mathrm{s}, \mathrm{NH}), 14.03(\mathrm{H}, \mathrm{s}, \mathrm{NH}) ;{ }^{13} \mathrm{C}-\mathrm{NMR}\left(\mathrm{CDCl}_{3}\right) \delta: 12.46,14.36,14.41,14.57(4 \mathrm{C}$, $\left.4 \mathrm{CH}_{3}\right), 60.93,63.12\left(2 \mathrm{C}, 2 \mathrm{CH}_{2}\right), 117.24,125.01,130.30,146.23$ (4C, thiophene carbons) $151.40(\mathrm{CO})$, $165.68(\mathrm{CO}), 173.16(\mathrm{C}=\mathrm{S})$.

2-(Ethoxycarbonylamino-carbothioyl)amino-7-benzyl-5,6,7,8-tetrahydro-3H-pyredo-[4 ; 3 :4,5] thiophene-3-carboxylic acid ethyl ester (10d). Light yellow powder, m.p. 205-207 ${ }^{\circ} \mathrm{C}$ (ethanol and DMF); yield 91\%; IR ( $\left.\mathrm{cm}^{-1}\right): 3,064(\mathrm{NH}), 3,024,1,728$ (ester $\left.\mathrm{CO}\right), 1,683$ (amide $\left.\mathrm{CO}\right), 1,564,1,525(\mathrm{C}=\mathrm{C}$ ), $1,110(\mathrm{C}=\mathrm{S}), 1,213(\mathrm{C}-\mathrm{O}) ;{ }^{1} \mathrm{H}-\mathrm{NMR}\left(\mathrm{DMSO}_{6}\right) \delta: 1.33\left(3 \mathrm{H}, \mathrm{t}, J=7.4 \mathrm{~Hz}, \mathrm{CH}_{3}\right), 1.36(3 \mathrm{H}, \mathrm{t}, J=7.4$ $\left.\mathrm{Hz}, \mathrm{CH}_{3}\right), 4.35\left(2 \mathrm{H}, \mathrm{q}, J=7.4 \mathrm{~Hz}, \mathrm{CH}_{2}\right), 4.43\left(2 \mathrm{H}, \mathrm{q}, J=7.4 \mathrm{~Hz}, \mathrm{CH}_{2}\right), 2.79\left(2 \mathrm{H}, \mathrm{m}, \mathrm{CH}_{2}\right), 2.94(2 \mathrm{H}$, m, $\left.\mathrm{CH}_{2}\right), 3.57\left(2 \mathrm{H}, \mathrm{s}, \mathrm{CH}_{2}\right), 3.71\left(2 \mathrm{H}, \mathrm{s}, \mathrm{CH}_{2}\right), 8.14(\mathrm{NH}-\mathrm{CO}), 14.09(\mathrm{NH}) ;{ }^{13} \mathrm{C}-\mathrm{NMR}\left(\mathrm{DMSO}-\mathrm{d}_{6}\right) \delta$ : $14.37\left(\mathrm{CH}_{3}\right), 14.44\left(\mathrm{CH}_{3}\right), 60.92$ and $63.19\left(2 \mathrm{CH}_{2}-\mathrm{O}\right), 27.01,50.32,51.36,62.16\left(\mathrm{CH}_{2}-\mathrm{N}\right), 115.61$, 125.48, 130.35, 147.91 (4C, thiophene carbons), 127.37, 128.46 (2C), 129.24 (2C), 138.12, 151.42 (amide CO), 165.52 (ester CO), $173.36(\mathrm{C}=\mathrm{S})$.

\subsection{General procedure for the synthesis of $\mathbf{1 1 a}, \mathbf{c}, \mathbf{d}$}

These susbstances were prepared according to a method reported in the literature [23]. Compound 10 (1 mmol) was dissolved in solution of sodium ethoxide $(230 \mathrm{mg}$ sodium and $15 \mathrm{~mL}$ of absolute ethanol) and the solution was heated under reflux for $30 \mathrm{~min}$. The solvent was then evaporated under vacuum, some water was added to the residue, and the $\mathrm{pH}$ of the mixture was adjusted to 4 with hydrochloric acid. The product that separated was collected and crystallized from a suitable solvent.

2-Thioxo-2,3,5,6,7,8-hexahydro-1H-benzo[4,5]thieno[2,3-d]pyrimidin-4-one (11a). White powder, m.p. $275-277^{\circ} \mathrm{C}$ (ethanol); yield 88\%; IR $\left(\mathrm{cm}^{-1}\right)$ : 3, $157(\mathrm{NH}), 1,672(\mathrm{CO}), 1,577,1,556(\mathrm{C}=\mathrm{C}), 1,157$ $(\mathrm{C}=\mathrm{S}) ;{ }^{1} \mathrm{H}-\mathrm{NMR}\left(\mathrm{DMSO}_{-} \mathrm{d}_{6}\right) \delta: 1.78\left(4 \mathrm{H}, \mathrm{m}, 2 \mathrm{CH}_{2}\right), 2.70\left(2 \mathrm{H}, \mathrm{m}, \mathrm{CH}_{2}\right), 2.80\left(2 \mathrm{H}, \mathrm{m}, \mathrm{CH}_{2}\right), 12.34$ (s, $\mathrm{NH}), 13.36(\mathrm{~s}, \mathrm{NH}) ;{ }^{13} \mathrm{C}-\mathrm{NMR}\left(\mathrm{DMSO}_{-} \mathrm{d}_{6}\right) \delta: 22.13,23.08,24.50,25.47\left(4 \mathrm{C}, 4 \mathrm{CH}_{2}\right), 117.04,128.78$, 131.43, 150.54 (4C, thiophene carbons), $157.58(\mathrm{CO}), 173.40(\mathrm{C}=\mathrm{S})$;

5,6-Dimethyl-2-thioxo-2,3-dihydro-1H-thieno[2,3-d]pyrimidin-4-one (11c). Light yellow powder, m.p. 205-207 ${ }^{\circ} \mathrm{C}$ (ethanol); yield 82\%; IR $\left(\mathrm{cm}^{-1}\right): 3,427,3,404(\mathrm{NH}), 1,664(\mathrm{CO}), 1,604,1,556(\mathrm{C}=\mathrm{C}), 1,174$ $(\mathrm{C}=\mathrm{S}) ;{ }^{1} \mathrm{H}-\mathrm{NMR}\left(\mathrm{DMSO}_{6}\right) \delta: 2.20\left(3 \mathrm{H}, \mathrm{s}, \mathrm{CH}_{3}\right), 2.24\left(3 \mathrm{H}, \mathrm{s}, \mathrm{CH}_{3}\right), 12.26(\mathrm{~s}, \mathrm{NH}), 13.26(\mathrm{~s}, \mathrm{NH}) ;{ }^{13} \mathrm{C}-$ NMR (DMSO-d $\left.{ }_{6}\right) \delta: 12.49,13.01\left(2 \mathrm{C}, 2 \mathrm{CH}_{3}\right), 117.71,125.68,129.21,149.79$ (4C, thiophene carbons), $157.71(\mathrm{CO}), 173.16(\mathrm{C}=\mathrm{S})$. 
7-Benzyl-2-thioxo-2,3,5,6,7,8-hexahydro-1H-pyrido[4 ,3 :4,5] thieno[2,3-d]pyrimidin-4-one

(11d).

Orange powder, m.p. $>300{ }^{\circ} \mathrm{C}$ (ethanol and DMF); yield 20\%; IR $\left(\mathrm{cm}^{-1}\right): 3,350,3,180(\mathrm{NH}), 3,076$,

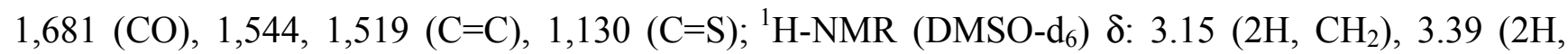
$\mathrm{CH}_{2}$ ), 4.24 (2H, $\left.\mathrm{CH}_{2}\right), 4.43$ (2H, s, $\left.\mathrm{CH}_{2}-\mathrm{Ph}\right), 7.26-7.38$ (5H, m, Ar-H), 11.40 (br. s, NH), 12.50 (s, NH).

\subsection{Synthesis of $\mathbf{1 2}$ and $\mathbf{1 3}$}

To a solution of $11 \mathrm{a}(1.19 \mathrm{~g}, 5 \mathrm{mmol})$ was added aroyl hydrazine $(5 \mathrm{mmol})$ in $n$-butanol $(15 \mathrm{~mL})$ and the mixture was heated under reflux for $20 \mathrm{~h}$. The solid obtained was cooled, collected and recrystallized from butanol.

1-(4-Chlorophenyl)-6, 7,8,9-tetrahydro-4H-benzo[4,5]thieno[2,3-d][1,2,4]triazolo[3,4-b]-pyrimidin-5one (12): Fine yellow crystals, m.p. $218-219{ }^{\circ} \mathrm{C}$; yield $86 \%$; IR $\left(\mathrm{cm}^{-1}\right): 3,253(\mathrm{NH}), 3,035(\mathrm{Ar}-\mathrm{CH})$, 1,656 (CO), 1,600 (C=N), 1,566 (C=C); ${ }^{1} \mathrm{H}-\mathrm{NMR}\left(\mathrm{DMSO}_{6}\right) \delta: 1.70\left(4 \mathrm{H}, 2 \mathrm{CH}_{2}\right), 2.63\left(2 \mathrm{H}, \mathrm{m}, \mathrm{CH}_{2}\right)$, $2.73\left(2 \mathrm{H}, \mathrm{m}, \mathrm{CH}_{2}\right), 7.52\left(2 \mathrm{H}, \mathrm{d}, J=8.8 \mathrm{~Hz}, \mathrm{C}_{6} \mathrm{H}_{4}\right), 7.82\left(2 \mathrm{H}, \mathrm{d}, J=8.8 \mathrm{~Hz}, \mathrm{C}_{6} \mathrm{H}_{4}\right), 9.87$ (br. s, NH); ${ }^{13} \mathrm{C}-\mathrm{NMR}\left(\mathrm{DMSO}-\mathrm{d}_{6}\right.$ ) $\delta: 22.05,22.00,24.42,25.39$ (aliphatic carbons), 116.97, 128.70, 131.36, 150.56 (4C, thiophene carbons), 128.96 (2C), 129.41 (2C), 136.43 (C-Cl), $132.56\left(\mathrm{C}, \mathrm{Ar}-\mathrm{C}^{1}\right), 157.82$ $(\mathrm{C}=\mathrm{N}), 165.34(\mathrm{C}=\mathrm{N}), 173.31(\mathrm{C}=\mathrm{O})$; MS: $\left.m / z 356\left(\mathrm{M}^{+}, 1.1 \%\right), 220\left(\mathrm{M}^{+}-\mathrm{Cl}_{-} \mathrm{C}_{6} \mathrm{H}_{4}-\mathrm{CN}\right)+\mathrm{H}, 9 \%\right), 193$ $\left(\mathrm{M}^{+}-\mathrm{Cl}-\mathrm{C}_{6} \mathrm{H}_{4}-\mathrm{CN}, \mathrm{C}_{2} \mathrm{H}_{4}+2 \mathrm{H}, 13 \%\right)$.

1-(3-Nitrophenyl)-6, 7,8,9-tetrahydro-4H-benzo[4,5]thieno[2,3-d] [1,2,4]triazolo[3,4-b]-pyrimidin-5one (13): Fine yellow powder, m.p. $234-235{ }^{\circ} \mathrm{C}$; Yield 79\%; IR $\left(\mathrm{cm}^{-1}\right)$ : 3,251 (NH), 3,107-3,041, $\left.1,687(\mathrm{CO}), 1,650(\mathrm{C}=\mathrm{N}), 1,579(\mathrm{C}=\mathrm{C}), 1,523,1,346(\mathrm{~N}=\mathrm{O}) ;{ }^{1} \mathrm{H}-\mathrm{NMR}\left(\mathrm{DMSO}_{\mathrm{d}}\right){ }\right)$ : $1.70(4 \mathrm{H}$, $\left.2 \mathrm{CH}_{2}\right), 2.60\left(2 \mathrm{H}, \mathrm{m}, \mathrm{CH}_{2}\right), 2.71\left(2 \mathrm{H}, \mathrm{m}, \mathrm{CH}_{2}\right), 7.73\left(1 \mathrm{H}, \mathrm{t}, J=8 \mathrm{~Hz}, \mathrm{Ar}-{ }^{5} \mathrm{CH}-m\right), 8.22(1 \mathrm{H}, \mathrm{d}$, $\left.J=6.0 \mathrm{~Hz}, \mathrm{Ar}-{ }^{6} \mathrm{CH}-o\right), 8.33\left(1 \mathrm{H}, \mathrm{d}, J=6.0 \mathrm{~Hz}, \mathrm{Ar}-{ }^{4} \mathrm{CH}-p\right), 8.60\left(1 \mathrm{H}, \mathrm{s}, \mathrm{Ar}-{ }^{2} \mathrm{CH}-o\right), 12.20(\mathrm{~s}, \mathrm{NH}) ;{ }^{13} \mathrm{C}-$ NMR (DMSO- $\mathrm{d}_{6}$ ) $\delta: 21.24,22.37,23.78,24,73$ (4C, aliphatic carbons), 116.36, 128.01, 130.77, 150.00 (4C, thiophene carbons), 121.66, 125.45, 129.97, 133.10 (4C, Ar-CH), 134.71 (C), 147.78 (C$\left.\mathrm{NO}_{2}\right), 156.81(\mathrm{C}=\mathrm{N}), 163.47(\mathrm{C}=\mathrm{N}), 172.86(\mathrm{C}=\mathrm{O})$; MS: $\mathrm{m} / z 367\left(\mathrm{M}^{+}, 87 \%\right), 221\left(\mathrm{M}^{+}-\left(\mathrm{N}=\mathrm{C}-\mathrm{C}_{6} \mathrm{H}_{3^{-}}\right.\right.$ $\left.\left.\mathrm{NO}_{2}\right)+2 \mathrm{H}, 24 \%\right), 207\left(\left(\mathrm{M}^{+}-\left[\left(\mathrm{N}=\mathrm{C}-\mathrm{C}_{6} \mathrm{H}_{3}-\mathrm{NO}_{2}\right)+2 \mathrm{H}\right]-\mathrm{NH}_{2}, 20 \%\right)\right.$.

\subsection{2-Hydrazino-5, 6, 7,8-tetrahydro-3H-benzo[4,5]thieno[2,3-d]pyrimidin-4-one (14)}

A mixture of 11a $(950 \mathrm{mg}, 4 \mathrm{mmol})$ and $99 \%$ hydrazine hydrate $(4 \mathrm{~mL}, 80 \mathrm{mmol})$ in pyridine $(20 \mathrm{~mL})$ was heated under reflux for $15 \mathrm{~h}$. The mixture was evaporated under reduced pressure and the residue was treated with ethanol. The solid product was filtered and washed several times with ethanol to give colorless crystals, m.p. $265-267{ }^{\circ} \mathrm{C}$; yield 83\%; IR $\left(\mathrm{cm}^{-1}\right): 3,319,3,265\left(\mathrm{NH}, \mathrm{NH}_{2}\right), 1,658$ (CO), 1,597 (C=C); ${ }^{1} \mathrm{H}-\mathrm{NMR}\left(\mathrm{DMSO}_{-} \mathrm{d}_{6}\right) \delta: 1.73\left(4 \mathrm{H}, \mathrm{m}, 2 \mathrm{CH}_{2}\right), 2.50\left(2 \mathrm{H}, \mathrm{m}, \mathrm{CH}_{2}\right), 2.75(2 \mathrm{H}, \mathrm{m}$, $\mathrm{CH}_{2}$ ), $8.20(\mathrm{NH}), \mathrm{NH}_{2}$ not observed; ${ }^{13} \mathrm{C}-\mathrm{NMR}\left(\mathrm{DMSO}_{\mathrm{d}}\right)$ ) $8: 22.49,23.30,24.73,25.84$ (aliphatic carbons), 114.25, 125.12, 130.60, 155.67 (thiophene carbons), $158.36(\mathrm{C}=\mathrm{N}), 167.14(\mathrm{CO})$ [13]. 
A mixture of 14 (1.18 g, $5 \mathrm{mmol})$ and excess triethyl orthoformate $(10 \mathrm{~mL})$ was heated under reflux with stirring for $2 \mathrm{~h}$. The excess of the orthoformate was removed under reduced pressure. The formed solid was collected, washed with ethanol, dried and recrystallized from ethanol to give a yellow powder, m.p. $277-279^{\circ} \mathrm{C}$; yield 84\%; IR $\left(\mathrm{cm}^{-1}\right)$ : 3, $118(\mathrm{NH}), 3,039,1,672(\mathrm{CO}), 1,608(\mathrm{C}=\mathrm{N}), 1,554$ $(\mathrm{C}=\mathrm{C}) ;{ }^{1} \mathrm{H}-\mathrm{NMR}\left(\mathrm{DMSO}_{6}\right): \delta 1.77\left(4 \mathrm{H}, 2 \mathrm{CH}_{2}\right), 2.62\left(2 \mathrm{H}, \mathrm{CH}_{2}\right), 2.83\left(2 \mathrm{H}, \mathrm{CH}_{2}\right), 9.01(1 \mathrm{H}, \mathrm{s}, \mathrm{CH})$, $14.04(1 \mathrm{H}, \mathrm{s}, \mathrm{NH}) ;{ }^{13} \mathrm{C}-\mathrm{NMR}\left(\mathrm{DMSO}_{6}\right) \delta: 22.40,23.17,24,91,25.94$ (4C, aliphatic carbons), 112.08, 126.64, 130.19, 148.87 (4C, thiophene carbons), $132.50(\mathrm{CH}$, triazole), $151.93(\mathrm{C}=\mathrm{N}), 167.82(\mathrm{C}=\mathrm{O})$; MS: $m / z: 246\left(\mathrm{M}^{+}, 87 \%\right), 245\left(\mathrm{M}^{+}-\mathrm{H}, 43 \%\right), 195\left(\mathrm{M}^{+}-\mathrm{HCN}, \mathrm{C}_{2} \mathrm{H}_{4}+4 \mathrm{H}, 37 \%\right)$.

\subsection{3-Methyl-5,6,7,8-tetrahydro-1H,2H-benzo[4,5]thieno[2,3-d][1,2,4]triazolo[3,4-a]-pyrimidin-4-} one (16)

A mixture of $14(1.18 \mathrm{~g}, 5 \mathrm{mmol})$ and excess triethyl orthoacetate $(10 \mathrm{~mL})$ was heated under reflux with stirring for $2 \mathrm{~h}$. The solvent was removed under reduced pressure, The formed solid was collected washed with ethanol, dried and recrystallized from ethanol, give a white powder, m.p. 292-294 ${ }^{\circ} \mathrm{C}$; yield 79\%; IR $\left(\mathrm{cm}^{-1}\right)$; 3,232 (NH), 1,714 (CO), 1,701 (C=N), 1,620 (C=C); ${ }^{1} \mathrm{H}-\mathrm{NMR}\left(\mathrm{DMSO}-\mathrm{d}_{6}\right): \delta$ $2.98\left(3 \mathrm{H}, \mathrm{s}, \mathrm{CH}_{3}\right), 1.68\left(4 \mathrm{H}, 2 \mathrm{CH}_{2}\right), 2.68\left(2 \mathrm{H}, \mathrm{CH}_{2}\right), 2.76\left(2 \mathrm{H}, \mathrm{CH}_{2}\right), 12.30(1 \mathrm{H}, \mathrm{s}, \mathrm{NH}) ; \mathrm{MS} m / z: 260$ $\left(\mathrm{M}^{+}, 100 \%\right), 245\left(\mathrm{M}^{+}-\mathrm{CH}_{3}, 15 \%\right), 232\left(\mathrm{M}^{+}-\left(\mathrm{C}_{2} \mathrm{H}_{4}\right), 57 \%\right)$.

\subsection{3-Thioxo-5, 6, 7,8-tetrahydro-1H,2H-benzo[4,5] thieno[2,3-d][1,2,4]triazolo[3,4-a]-pyrimidin-4-} one (17)

A mixture of 14 (1.18 g, $5 \mathrm{mmol})$ in pyridine $(15 \mathrm{ml})$ and carbon disulfide $(380 \mathrm{mg}, 5 \mathrm{mmol})$ was heated under reflux for $5 \mathrm{~h}$. After cooling, the obtained solid was collected and recrystallized from acetic acid to give a white powder, m.p. $>300{ }^{\circ} \mathrm{C}$; yield $81 \%$; IR $\left(\mathrm{cm}^{-1}\right): 3,105(\mathrm{br}, \mathrm{NH}), 2,945,1,664$ (CO); ${ }^{1} \mathrm{H}-\mathrm{NMR}\left(\mathrm{DMSO}_{6}\right) \delta: 1.75\left(4 \mathrm{H}, 2 \mathrm{CH}_{2}\right), 2.73\left(2 \mathrm{H}, \mathrm{br}, \mathrm{CH}_{2}\right), 2.83\left(2 \mathrm{H}, \mathrm{br}, \mathrm{CH}_{2}\right), 12.57$ (br. s, $\mathrm{NH}), 13.86$ (br. s, NH); ${ }^{13} \mathrm{C}-\mathrm{NMR}\left(\mathrm{DMSO}_{-} \mathrm{d}_{6}\right) \delta: 22.19,22.92,24.41,25.16$ (4C, aliphatic carbons), 118.33, 130.93, 131.72, $139.32(4 \mathrm{C}$, thiophene carbons), $144.98(\mathrm{C}=\mathrm{N}), 157.71(\mathrm{C}=\mathrm{O}), 158.81(\mathrm{C}=\mathrm{S})$; MS: $m / z 278\left(\mathrm{M}^{+}, 100 \%\right), 250\left(\mathrm{M}^{+}-\mathrm{C}_{2} \mathrm{H}_{4}, 43 \%\right), 245\left(\mathrm{M}^{+}-\mathrm{SH}, 31 \%\right)$.

\subsection{Synthesis of disubstituted thienyl-2-thioureides $\mathbf{2 0 - 2 3}$}

\subsubsection{Method A}

2-Amino-3-ethoxycarbonyl thiophene 1a,d (100 mmol) was dissolved in hot ethanol $(100 \mathrm{~mL})$ and phenyl (or $p$-clorophenyl) isothiocyanate $(110 \mathrm{mmol})$ was added dropwise with stirring. The reaction mixture was heated under reflux on water bath for $2 \mathrm{~h}$, then left to cool overnight and the separated crude solid was filtered, washed with ethanol, and recrystallized from ethanol [12]. 


\subsubsection{Method-B}

A mixture of 1a,d (20 mmol) and phenyl (or $p$-clorophenyl) isothiocyanate $(20 \mathrm{mmol})$ placed in a $50 \mathrm{~mL}$ conical flask covered with a funnel glass and then irradiated with microwaves $(950 \mathrm{~W})$ for 35-40 seconds. The cold reaction mixture was then treated with ethanol and the solid product was filtered off and recrystallized from ethanol [13].

2-(3-Phenylthioureido)-4,5,6,7-tetrahydrobenzo[b]thiophene-3-carboxylic acid ethyl ester (20). Fine colorless needles, m.p. $187-189{ }^{\circ} \mathrm{C}$; yield: $(78 \%)^{\mathrm{A}},(89 \%)^{\mathrm{B}}$; IR $\left(\mathrm{cm}^{-1}\right): 3,196(\mathrm{NH}), 3,032(\mathrm{Ar}-\mathrm{CH})$, 1,656 (CO), 1,554 (C=C), 1,195 (C=S); ${ }^{1} \mathrm{H}-\mathrm{NMR}\left(\mathrm{CDCl}_{3}\right) \delta: 0.97\left(3 \mathrm{H}, J=7.3 \mathrm{~Hz}, \mathrm{t}, \mathrm{CH}_{2} \mathrm{CH}_{3}\right), 1.43$ $\left(4 \mathrm{H}, \mathrm{m}, 2 \mathrm{CH}_{2}\right), 2.27\left(2 \mathrm{H}, \mathrm{m}, \mathrm{CH}_{2}\right), 2.41\left(2 \mathrm{H}, \mathrm{m}, \mathrm{CH}_{2}\right), 3.87\left(2 \mathrm{H}, J=7.3 \mathrm{~Hz}, \mathrm{q}, \mathrm{CH}_{2}-\mathrm{CH}_{3}\right), 6.89$ (t, $J=7.32 \mathrm{~Hz}, \operatorname{Ar}-\mathrm{CH}(p)), 7.05(2 \mathrm{H}, \mathrm{t}, J=7.32 \mathrm{~Hz}, \operatorname{Ar}-\mathrm{CH}(m)), 7.11(2 \mathrm{H}, \mathrm{d}, J=8.08 \mathrm{~Hz}, \operatorname{Ar}-\mathrm{CH}(o))$, $10.03(\mathrm{~s}, \mathrm{NH}), 11.70(\mathrm{~s}, \mathrm{NH}) ;{ }^{13} \mathrm{C}-\mathrm{NMR}\left(\mathrm{CDCl}_{3}\right) \delta: 14.25\left(\mathrm{CH}_{3}\right), 22.92,23.02,24,09,26.17$ (aliphatic carbons); $60.14\left(\mathrm{O}_{-} \mathrm{CH}_{2}\right), 112.01,125.89,130.35,150.17$ (4C, thiophene carbons); 124.51, 125.89(2C), $128.94(2 \mathrm{C}), 137.81$ (aromatic carbons), $166.13(\mathrm{C}=\mathrm{O}) ; 175.99(\mathrm{C}=\mathrm{S}) ; \mathrm{MS}: \mathrm{m} / z: 360\left(\mathrm{M}^{+}\right.$, $20 \%), 326\left(\mathrm{M}^{+}-\mathrm{SH}, \mathrm{H}, 17 \%\right), 225\left(\mathrm{M}^{+}-\left(\mathrm{SH}, \mathrm{H}, \mathrm{C}_{6} \mathrm{H}_{5}, \mathrm{CN}\right)+2 \mathrm{H}, 33 \%\right)$.

2-(3-(4-Chlorophenyl)thioureido)-4,5,6,7-tetrahydrobenzo[b]thiophene-3-carboxylic acid ethyl ester (21). Yellow needles, m.p. $198-199^{\circ} \mathrm{C}$; yield $(71 \%)^{\mathrm{A}},(88 \%)^{\mathrm{B}}$; IR $\left(\mathrm{cm}^{-1}\right): 3,141(\mathrm{NH}), 3,078(\mathrm{Ar}-\mathrm{CH})$, 1,652 (CO), 1,558 (C=C), 1,195 (C=S); ${ }^{1} \mathrm{H}-\mathrm{NMR}\left(\mathrm{CDCl}_{3}\right) \delta: 1.25\left(3 \mathrm{H}, \mathrm{t}, \mathrm{CH}_{2} \mathrm{CH}_{3}\right), 1.75(4 \mathrm{H}, \mathrm{m}$, $\left.2 \mathrm{CH}_{2}\right), 2.60\left(2 \mathrm{H}, \mathrm{m}, \mathrm{CH}_{2}\right), 2.70\left(2 \mathrm{H}, \mathrm{m}, \mathrm{CH}_{2}\right), 4.15\left(2 \mathrm{H}, \mathrm{q}, \mathrm{CH}_{2} \mathrm{CH}_{3}\right), 7.28(2 \mathrm{H}, \mathrm{d}, J=8.8 \mathrm{~Hz}, \mathrm{Ar}-\mathrm{CH})$, 7.41 (2, d, $J=8.8 \mathrm{~Hz}, \mathrm{Ar}-\mathrm{CH}), 7.92$ (br. s, NH), $12.25(1 \mathrm{H}, \mathrm{s}, \mathrm{NH}) ;{ }^{13} \mathrm{C}-\mathrm{NMR}\left(\mathrm{CDCl}_{3}\right) \delta: 14.12\left(\mathrm{CH}_{3}\right)$, 22.92, 23.02, 24.40, 26.38 (4C, aliphatic carbons), $60.65\left(\mathrm{O}-\mathrm{CH}_{2}\right), 113.23,127.28,133.18,149.81$ (thiophene carbons), $126.80(2 \mathrm{C}), 130.13(2 \mathrm{C}), 130.96,134.66$ (phenyl carbons), $166.68(\mathrm{C}=\mathrm{O})$, $176.06(\mathrm{C}=\mathrm{S})$; MS: $m / z 394\left(\mathrm{M}^{+}, 26 \%\right) 396\left(\mathrm{M}^{+}+2,15 \%\right), 225\left(\mathrm{M}^{+}-\left(\mathrm{OCH}_{2} \mathrm{CH}_{3}, \mathrm{NH}-\mathrm{C}_{6} \mathrm{H}_{4} \mathrm{Cl}\right)+2 \mathrm{H}\right.$, $39 \%), 179\left(\mathrm{M}^{+}-\left(\mathrm{OCH}_{2} \mathrm{CH}_{3}, \mathrm{NHCSNH}-\mathrm{C}_{6} \mathrm{H}_{4} \mathrm{Cl}\right), 100 \%\right)$.

6-Benzyl-2-(3-phenylthioureido)-4,5,6,7-tetrahydrothieno[2,3-c]pyridine-3-carboxylic acid ethyl ester (22). Orange needles, m.p. $274-276^{\circ} \mathrm{C}$; yield $(64 \%)^{\mathrm{A}},(77 \%)^{\mathrm{B}}$; IR $\left(\mathrm{cm}^{-1}\right): 3,196(\mathrm{br}, \mathrm{NH}), 3,032(\mathrm{Ar}-$ $\mathrm{CH}), 1,656(\mathrm{CO}), 1,554(\mathrm{C}=\mathrm{C}), 1,197(\mathrm{C}=\mathrm{S}) ;{ }^{1} \mathrm{H}-\mathrm{NMR}\left(\mathrm{CDCl}_{3}\right) \delta: 1.21\left(3 \mathrm{H}, J=7.3 \mathrm{~Hz}, \mathrm{t}, \mathrm{CH}_{2} \mathrm{CH}_{3}\right)$, $2.93\left(4 \mathrm{H}, \mathrm{m}, 2 \mathrm{CH}_{2}\right), 3.37\left(4 \mathrm{H}\right.$, br. s, $\left.2 \mathrm{CH}_{2}\right), 4.12\left(2 \mathrm{H}, J=7.3 \mathrm{~Hz}, \mathrm{q}, \mathrm{CH}_{2} \mathrm{CH}_{3}\right), 7.32\left(5 \mathrm{H}, \mathrm{m}, \mathrm{C}_{6} \mathrm{H}_{5}\right)$, $7.46\left(5 \mathrm{H}, \mathrm{m}, \mathrm{C}_{6} \mathrm{H}_{5}\right), 7.95$ (s, NH), 12.11 (s, NH); MS: m/z $450\left(\mathrm{M}^{+}-\mathrm{H}, 16 \%\right), 91\left(\left(\mathrm{Ph}_{-} \mathrm{CH}_{2}\right), 100 \%\right)$.

6-Benzyl-2-(3-(4-chlorophenyl)thioureido)-4,5,6,7-tetrahydro thieno [2,3-c]pyridine-3-carboxylic acid ethyl ester (23). Yellow needles, m.p. 259-261 ${ }^{\circ} \mathrm{C}$; yield $(54 \%)^{\mathrm{A}},(69 \%)^{\mathrm{B}}$; IR $\left(\mathrm{cm}^{-1}\right): 3,176(\mathrm{NH})$, 3,053, 3,031 (Ar-CH), 1,652 (CO), 1,558 (C=C), 1,197 (C=S); ${ }^{1} \mathrm{H}-\mathrm{NMR}\left(\mathrm{CDCl}_{3}\right) \delta: 1.24(3 \mathrm{H}, \mathrm{t}$, $\left.J=7.36 \mathrm{~Hz}, \mathrm{CH}_{2} \mathrm{CH}_{3}\right), 2.85\left(4 \mathrm{H}, \mathrm{m}, 2 \mathrm{CH}_{2}\right), 3.57\left(2 \mathrm{H}, \mathrm{s}, \mathrm{CH}_{2}\right), 3.74\left(2 \mathrm{H}, \mathrm{s}, \mathrm{CH}_{2}\right), 4.17(2 \mathrm{H}, \mathrm{q}$, $\left.J=7.36 \mathrm{~Hz}, \mathrm{CH}_{2} \mathrm{CH}_{3}\right), 7.22(2 \mathrm{H}, \mathrm{d}, J=8.8 \mathrm{~Hz}, \mathrm{Ar}-\mathrm{CH}), 7.38(2 \mathrm{H}, \mathrm{d}, J=8.8 \mathrm{~Hz}, \mathrm{Ar}-\mathrm{CH}), 7.24-7.39$ $\left(5 \mathrm{H}, \mathrm{m}, \mathrm{C}_{6} \mathrm{H}_{5}\right), 8.37$ (br. s, NH), $12.15(\mathrm{~s}, \mathrm{NH}) ;{ }^{13} \mathrm{C}-\mathrm{NMR}\left(\mathrm{CDCl}_{3}\right) \delta: 14.25\left(\mathrm{CH}_{3}\right), 26.46,50.13,51.02$, $61.85\left(4 \mathrm{C}, \mathrm{CH}_{2}\right) ; 60.77\left(\mathrm{O}-\mathrm{CH}_{2}\right), 112.56,127.12,133.19,150.65$ (4C, thiophene ring); 123.90, 126.85 (2C), $130.09(2 \mathrm{C}), 134.62\left(\mathrm{C}_{6} \mathrm{H}_{5}\right), 126.87(2 \mathrm{C}), 128.52(2 \mathrm{C}), 129.17,129.41\left(p-\mathrm{Cl}-\mathrm{C}_{6} \mathrm{H}_{4}\right), 166.35$ $(\mathrm{C}=\mathrm{O}), 176.03(\mathrm{C}=\mathrm{S})$; MS: $m / z 485\left(\mathrm{M}^{+}, 0.02 \%\right), 316\left(\mathrm{M}^{+}-\left(\mathrm{NH}-\mathrm{C}_{6} \mathrm{H}_{4} \mathrm{Cl}_{2} \mathrm{OCH}_{2} \mathrm{CH}_{3}\right)+\mathrm{H}, 9 \%\right), 168$ $\left(\mathrm{M}^{+}-\left(\mathrm{NH}-\mathrm{C}_{6} \mathrm{H}_{4} \mathrm{Cl}, \mathrm{OCH}_{2} \mathrm{CH}_{3}, \mathrm{CH}_{2} \mathrm{C}_{6} \mathrm{H}_{5}, \mathrm{NCH}_{2}, \mathrm{CO}\right), 80 \%\right)$. 


\subsection{General procedure for the preparation of the monopotassium salts of $\mathbf{2 4}$ and $\mathbf{2 5}$}

A mixture of the compounds 21, $22(13.5 \mathrm{mmol})$ and potassium hydroxide (760 $\mathrm{mg}, 13.5 \mathrm{mmol})$ in absolute ethanol $(55 \mathrm{~mL})$ was heated under reflux with stirring for $1 \mathrm{~h}$. The suspension was filtered while hot, and the solid was washed with hot absolute ethanol to give 24) [13] and 25 which were both used without any further purification.

\subsection{General procedure for the preparation of $\mathbf{1 8 a , d}$}

\subsubsection{Method A}

A suspension of potassium salts of $\mathbf{2 4 , 2 5}$ in water $(50 \mathrm{~mL})$ was acidified with concentrated hydrochloric acid and stirred at room temperature for $30 \mathrm{~min}$. The solid was collected by filtration, washed with water and recrystallized from the suitable solvent to give 18a,d.

\subsubsection{Method B: Synthesis of $\mathbf{1 8 d}$}

A mixture of $1 \mathbf{d}(3.16 \mathrm{~g}, 10 \mathrm{mmol})$ and phenyl isothiocyanate $(1350 \mathrm{mg}, 10 \mathrm{mmol})$ in acetonitrile $(30 \mathrm{~mL})$ was heated under reflux for $15 \mathrm{~h}$ in the presence of anhydrous potassium carbonate $(1.4 \mathrm{~g})$. The reaction mixture was then cooled, filtered, diluted with water $(10 \mathrm{~mL})$ and neutralized with $2 \mathrm{M}$ hydrochloric acid. The product obtained was filtered, washed with water, dried and recrystallized from acetic acid.

Monopotassium salt of 3-(4-chlorophenyl)-2-thioxo-2,3,5,6,7,8-hexahydro-1H-[4,5]thieno[2,3-d]pyrimidin-4-one (24) and its 2-thioxo derivative 18a. Yields 54\% (24) and 72\% (18a), recrystallized from ethanol to give a white powder, m.p. $>300^{\circ} \mathrm{C}$; IR $\left(\mathrm{cm}^{-1}\right): 3,130(\mathrm{NH}), 3,060,3,039,1,693$ (CO), 1,524, 1,490 (C=C); ${ }^{1} \mathrm{H}-\mathrm{NMR}\left(\mathrm{CDCl}_{3}\right) \delta: 1.85\left(4 \mathrm{H}, \mathrm{m}, 2 \mathrm{CH}_{2}\right), 2.75\left(2 \mathrm{H}, \mathrm{m}, \mathrm{CH}_{2}\right), 2.91\left(2 \mathrm{H}, \mathrm{m}, \mathrm{CH}_{2}\right)$, $7.34(2 \mathrm{H}, \mathrm{d}, J=8.8 \mathrm{~Hz}, \mathrm{Ar}-\mathrm{CH}), 7.55(2 \mathrm{H}, \mathrm{d}, J=8.8 \mathrm{~Hz}, \mathrm{Ar}-\mathrm{CH}), 7.26(1 \mathrm{H}, \mathrm{s}, \mathrm{NH})$; MS: $m / z 348\left(\mathrm{M}^{+}\right.$, $50 \%), 206\left(\mathrm{M}^{+}-\mathrm{C}_{6} \mathrm{H}_{5} \mathrm{Cl}, \mathrm{SH}+2 \mathrm{H}, 58 \%\right), 111\left(\mathrm{M}^{+}-\left(\mathrm{C}_{6} \mathrm{H}_{5} \mathrm{Cl}, \mathrm{SH}, 2 \mathrm{C}_{2} \mathrm{H}_{4}, \mathrm{~N},+3 \mathrm{H}\right), 56 \%\right)$.

Monopotassium salt of 7-benzyl-3-phenyl-2-thioxo-2,3,5,6,7,8-hexahydro-1H-pyrido[3 ‘4:4,5]thieno[2,3-d]-pyrimidin-4-one (25) and its 2-thioxo derivative 18d. Yields: $49 \%(\mathbf{2 5})$ and $59 \%{ }^{\mathrm{A}}, 46 \%{ }^{\mathrm{B}}$ (18d); Yellow powder, m.p. $273-274^{\circ} \mathrm{C}$ (acetic acid); IR ( $\left.\mathrm{cm}^{-1}\right)$ : 3,390 (NH), 3,061, 1,689 (CO), 1,523 $(\mathrm{C}=\mathrm{C})$; MS: $m / z 405\left(\mathrm{M}^{+}, 27 \%\right), 314\left(\mathrm{M}^{+}-\left(\mathrm{CH}_{2}-\mathrm{Ph}\right), 7 \%\right), 286\left(\mathrm{M}^{+}-\left(\mathrm{CH}_{2}-\mathrm{Ph}, \mathrm{N}-\mathrm{CH}_{2}\right), 29 \%\right)$.

\subsection{7-Benzyl-3-phenyl-2-hydrazino-5,6,7,8-tetrahydro-1H-pyrido[3 ‘, ‘:4,5]thieno[2,3-d]-pyrimidin- 4-one (19)}

A mixture of 18d (1.62 $\mathrm{g}, 4 \mathrm{mmol})$ and $99 \%$ hydrazine hydrate $(4 \mathrm{~mL}, 80 \mathrm{mmol})$ in pyridine $(20 \mathrm{~mL})$ was heated under reflux for $15 \mathrm{~h}$. The mixture was evaporated under reduced pressure and the residue was treated with ethanol. The solid product was filtered, washed with ethanol, dried and recrystallized from ethanol [13] to give an orange powder, m.p. 186-187 ${ }^{\circ} \mathrm{C}$; Yield 75\%; IR $\left(\mathrm{cm}^{-1}\right)$ : 3,311-3,145 (NH, NH$), 3,034,1,689(\mathrm{C}=\mathrm{O}), 1,546,1,504(\mathrm{C}=\mathrm{C}) ;{ }^{1} \mathrm{H}-\mathrm{NMR}\left(\mathrm{CDCl}_{3}\right) \delta: 2.82$ $\left(2 \mathrm{H}, \mathrm{m}, \mathrm{CH}_{2}\right), 2.99\left(2 \mathrm{H}, \mathrm{m}, \mathrm{CH}_{2}\right), 3.61\left(2 \mathrm{H}, \mathrm{s}, \mathrm{CH}_{2}\right), 3.72\left(2 \mathrm{H}, \mathrm{s}, \mathrm{CH}_{2}\right), 3.89\left(2 \mathrm{H}, \mathrm{s}, \mathrm{NH}_{2}\right), 5.40$ (s, $\mathrm{NH}), 7.22(2 \mathrm{H}, \mathrm{d}, J=8.8 \mathrm{~Hz}, \mathrm{Ar}-\mathrm{CH}), 7.38(2 \mathrm{H}, \mathrm{d}, J=8.8 \mathrm{~Hz}, \mathrm{Ar}-\mathrm{CH}), 7.23-7.58\left(6 \mathrm{H}, \mathrm{m}_{,} \mathrm{C}_{6} \mathrm{H}_{5}\right) ;{ }^{13} \mathrm{C}-$ 
NMR $\left(\mathrm{CDCl}_{3}\right) \delta: 25.68,49.97,51.61,62.10\left(4 \mathrm{C}, \mathrm{CH}_{2}\right), 115.39,125.48,133.65,152.68$ (4C, thiophene carbons), [127.36, 128.46 (2C), 128.78 (2C), 129.24 (2C), 129.95, 130.14, 130.69 (2C), 138.14 (12C, $\left.2 \mathrm{C}_{6} \mathrm{H}_{4}\right)$ ], $158.41(\mathrm{C}=\mathrm{N}), 164.85(\mathrm{C}=\mathrm{O})$; MS: $m / z 403\left(\mathrm{M}^{+}, 47 \%\right), 387\left(\mathrm{M}^{+}-\left(\mathrm{NH}_{2}\right), 20 \%\right), 312\left(\mathrm{M}^{+}-\mathrm{CH}_{2^{-}}\right.$ $\mathrm{Ph}, 8 \%), 284\left(\mathrm{M}^{+}-\mathrm{CH}_{2}-\mathrm{Ph}, \mathrm{N}-\mathrm{CH}_{2}, 66 \%\right)$.

\subsection{General procedure for the preparation of $\mathbf{2 6 - 2 9}$}

A mixture of thiouredo derivatives $\mathbf{2 0 - 2 3}(10 \mathrm{mmol})$ and hydrazine hydrate $(20 \mathrm{mmol})$ in ethanol $(100 \mathrm{~mL})$ was heated under reflux for 3-4 h. The solid that separated upon cooling was filtered, washed with water, dried and recrystallized from ethanol.

3-Amino-2-phenylamino-5,6,7,8-tetrahydro-3H-benzo[4,5]thieno[2,3-d]pyrimidin-4-one (26). Fine colorless crystals, m.p. 204-205 ${ }^{\circ} \mathrm{C}$; Yield 56\%; IR $\left(\mathrm{cm}^{-1}\right)$; 3,323, 3,309, 3,259 $\left(\mathrm{NH}_{2}, \mathrm{NH}\right), 3,034$, 1,672 (CO), 1,606, 1,544 (C=C); ${ }^{1} \mathrm{H}-\mathrm{NMR}\left(\mathrm{DMSO}_{\mathrm{d}}\right) \delta$ : $1.76\left(4 \mathrm{H}, \mathrm{m}, 2 \mathrm{CH}_{2}\right), 2.63\left(2 \mathrm{H}, \mathrm{m}, \mathrm{CH}_{2}\right), 2.82$ $\left(2 \mathrm{H}, \mathrm{m}, \mathrm{CH}_{2}\right), 5.59\left(2 \mathrm{H}, \mathrm{s}, \mathrm{NH}_{2}\right), 7.06(1 \mathrm{H}, \mathrm{t}, J=7.3 \mathrm{~Hz}, \mathrm{Ar}-\mathrm{CH}(p)), 7.34(2 \mathrm{H}, \mathrm{t}, J=7.3 \mathrm{~Hz}, \mathrm{Ar}-$ $\mathrm{CH}(m)), 7.74(2 \mathrm{H}, \mathrm{d}, J=8.08 \mathrm{~Hz}, \operatorname{Ar}-\mathrm{CH}(o)), 9.35(1 \mathrm{H}, \mathrm{s}, \mathrm{NH}) ;{ }^{13} \mathrm{C}-\mathrm{NMR}\left(\mathrm{DMSO}_{6}\right)$ ) $22.42,23.24$, 24.84, 25.77 (aliphatic carbons), 115.19, 127.48, 130.72, 149.30 (thiophene carbons), 121.39, 123.69, 129.14, 138.83 (phenyl carbons), $157.99(\mathrm{C}=\mathrm{N}), 166.13(\mathrm{C}=\mathrm{O})$; MS: $m / z 312\left(\mathrm{M}^{+}, 20 \%\right), 297\left(\mathrm{M}^{+}-\right.$ $\left.\left.\mathrm{NH}_{2}\right)+\mathrm{H}, 21 \%\right), 235\left(\mathrm{M}^{+}-\mathrm{Ph}, 36 \%\right), 221\left(\mathrm{M}^{+}-\mathrm{NH} \mathrm{Ph}+\mathrm{H}, 46 \%\right)$.

3-Amino-2-(4-chlorophenyl)amino-5, 6, 7,8-tetrahydro-3H-benzo[4,5]thieno [2,3-d]pyrimidin-4-one (27). colorless crystals, m.p. $166-167{ }^{\circ} \mathrm{C}$; Yield: 46\%; IR $\left(\mathrm{cm}^{-1}\right) ; 3,334,3,330,3,203\left(\mathrm{NH}_{2}, \mathrm{NH}\right)$, 3,099, 1,664 (CO), 1,587, 1,546 (C=C); ${ }^{1} \mathrm{H}-\mathrm{NMR}\left(\mathrm{DMSO}_{6}\right) \delta: 1.78\left(4 \mathrm{H}, \mathrm{m}, 2 \mathrm{CH}_{2}\right), 2.64(2 \mathrm{H}, \mathrm{m}$, $\left.\mathrm{CH}_{2}\right), 2.87\left(2 \mathrm{H}, \mathrm{m}, \mathrm{CH}_{2}\right), 4.57\left(2 \mathrm{H}, \mathrm{s}, \mathrm{NH}_{2}\right), 7.29(2 \mathrm{H}, \mathrm{d}, J=8.8 \mathrm{~Hz}, \mathrm{Ar}-\mathrm{CH}), 7.59(2 \mathrm{H}, \mathrm{d}, J=8.8 \mathrm{~Hz}$, $\mathrm{Ar}-\mathrm{CH}), 8.53$ (s, NH); ${ }^{13} \mathrm{C}-\mathrm{NMR}\left(\mathrm{DMSO}_{\mathrm{d}}\right.$ ) $\delta: 22.34,23.11,25.00,25.45$ (4C, aliphatic ring), 115.54, 128.56, 130.69, 147.15 (4C, thiophene ring); 121.14 (2C), 129.05 (2C), 129.15, 136.41 (aromatic carbons), $158.20(\mathrm{C}=\mathrm{N}), 163.09(\mathrm{C}=\mathrm{O})$; MS: $m / z 346\left(\mathrm{M}^{+}, 98 \%\right), 348\left(\mathrm{M}^{+}+1,54 \%\right), 312\left(\mathrm{M}^{+}-\mathrm{Cl}+\mathrm{H}\right.$, 14\%), $\left.330\left(\mathrm{M}^{+}-\mathrm{NH}_{2}, 15 \%\right), 331\left(\mathrm{M}^{+}-\mathrm{NH}_{2}+\mathrm{H}, 6 \%\right), 220\left(\mathrm{M}^{+}-\mathrm{NH}_{2}, \mathrm{Ph}-\mathrm{Cl}\right)+\mathrm{H}, 5 \%\right), 206\left(\mathrm{M}^{+}-\mathrm{NH}_{2}\right.$, $\mathrm{NH} \mathrm{Ph}-\mathrm{Cl})+2 \mathrm{H}, 38 \%), 179\left(\mathrm{M}^{+}-\mathrm{NH}_{2}, \mathrm{NH} \mathrm{Ph}-\mathrm{Cl}, \mathrm{HCN}+2 \mathrm{H}, 32 \%\right)$.

7-Benzyl-3-Amino-2-phenylamino-5, 6, 7,8-tetrahydro-3H-pyrido[3 4 $4: 4,5]$ thieno[2,3-d]pyrimidin-4one (28). Light yellow crystals, m.p. $150-152{ }^{\circ} \mathrm{C}$; Yield $32 \%$; IR $\left(\mathrm{cm}^{-1}\right): 3,317,3,300\left(\mathrm{NH}_{2}, \mathrm{NH}\right)$, 3,032 (Ar-CH), 1,691 (CO), 1,589, 1,543 (C=C); ${ }^{1} \mathrm{H}-\mathrm{NMR}\left(\mathrm{CDCl}_{3}\right) \delta: 2.78\left(2 \mathrm{H}, \mathrm{t}, \mathrm{CH}_{2}\right), 2.99(2 \mathrm{H}, \mathrm{t}$, $\left.\mathrm{CH}_{2}\right), 3.46\left(2 \mathrm{H}, \mathrm{s}, \mathrm{CH}_{2}\right), 3.68\left(2 \mathrm{H}, \mathrm{s}, \mathrm{CH}_{2}\right), 4.81\left(2 \mathrm{H}, \mathrm{s}, \mathrm{NH}_{2}\right), 7.07(1 \mathrm{H}, \mathrm{t}, J=7.3 \mathrm{~Hz}, \mathrm{Ar}-\mathrm{CH}(p)), 7.34$ $(2 \mathrm{H}, \mathrm{t}, J=8.08 \mathrm{~Hz}, \operatorname{Ar}-\mathrm{CH}(\mathrm{m})), 7.55(2 \mathrm{H}, \mathrm{d}, J=8.08 \mathrm{~Hz}, \operatorname{Ar}-\mathrm{CH}(o)), 7.40-7.35\left(5 \mathrm{H}, \mathrm{m}, \mathrm{C}_{6} \mathrm{H}_{5}\right), 8.42$ $(1 \mathrm{H}, \mathrm{s}, \mathrm{NH}) ;{ }^{13} \mathrm{C}-\mathrm{NMR}\left(\mathrm{CDCl}_{3}\right) \delta: 25.78,50.21,51.60,62.52$ (aliphatic carbons), 114.73, 127.48, 137.73, 147.52 (4C, thiophene carbons), [120.02 (2C), 123.63, 125.61, 128.51, 129.02 (2C), 129.44 (2C), $129.65(2 \mathrm{C}), 137.84$, aromatic carbons], $157.97(\mathrm{C}=\mathrm{N}), 163.66(\mathrm{C}=\mathrm{O})$; $\mathrm{MS}: \mathrm{m} / z 403\left(\mathrm{M}^{+}, 49 \%\right)$, $387\left(\mathrm{M}^{+}\left(-\mathrm{NH}_{2}\right), 14 \%\right), 311\left(\mathrm{M}^{+}-\left(\mathrm{C}_{6} \mathrm{H}_{5}, \mathrm{NH}\right), 16 \%\right), 284\left(\mathrm{M}^{+}-\left(\mathrm{CH}_{2} \mathrm{C}_{6} \mathrm{H}_{5}, \mathrm{~N}-\mathrm{CH}_{2}, 43 \%\right), 176\left(\mathrm{M}^{+}-\right.\right.$ $\left.\left(\mathrm{CH}_{2} \mathrm{C}_{6} \mathrm{H}_{5}, \mathrm{~N}-\mathrm{CH}_{2}, \mathrm{NH}_{2}, \mathrm{NH}-\mathrm{C}_{6} \mathrm{H}_{5}\right), 9 \%\right), 150\left(\mathrm{M}^{+}-\left(\mathrm{CH}_{2} \mathrm{C}_{6} \mathrm{H}_{5}, \mathrm{~N}-\mathrm{CH}_{2}, \mathrm{NH}_{2}, \mathrm{NH}-\mathrm{C}_{6} \mathrm{H}_{5}, \mathrm{HCN}\right)+(\mathrm{H})\right.$, $23 \%)$. 
7-Benzyl-3-Amino-2-(4-chlorophenyl)amino-5, 6, 7,8-tetrahydro-3H-pyrido [3 `4 :4,5]thieno[2,3-d] pyrimidin-4-one (29). Light yellow crystals, m.p. $192-194{ }^{\circ} \mathrm{C}$; Yield: $29 \%$; IR $\left(\mathrm{cm}^{-1}\right)$ : 3,450-3,313 (br, $\left.\mathrm{NH}_{2}\right), 3,201(\mathrm{NH}), 3,032(\mathrm{Ar}-\mathrm{CH}), 1,674(\mathrm{CO}), 1,537(\mathrm{C}=\mathrm{C}) ;{ }^{1} \mathrm{H}-\mathrm{NMR}\left(\mathrm{CDCl}_{3}\right) \delta: 2.78\left(2 \mathrm{H}, \mathrm{m}, \mathrm{CH}_{2}\right)$, $3.02\left(2 \mathrm{H}, \mathrm{m}, \mathrm{CH}_{2}\right), 3.44\left(2 \mathrm{H}, \mathrm{s}, \mathrm{CH}_{2}\right), 3.68\left(2 \mathrm{H}, \mathrm{s}, \mathrm{CH}_{2}\right), 4.86\left(2 \mathrm{H}, \mathrm{s}, \mathrm{NH}_{2}\right), 7.25(2 \mathrm{H}, \mathrm{d}$, $J=8.8 \mathrm{~Hz}, \mathrm{Ar}-\mathrm{CH}), 7.47(2 \mathrm{H}, \mathrm{d}, J=8.8 \mathrm{~Hz}, \mathrm{Ar}-\mathrm{CH}), 7.37-7.30\left(5 \mathrm{H}, \mathrm{m}, \mathrm{C}_{6} \mathrm{H}_{5}\right), 8.40(\mathrm{~s}, \mathrm{NH}) ;{ }^{13} \mathrm{C}-$ NMR $\left(\mathrm{CDCl}_{3}\right) \delta: 25.78,50.31,51.56,62.62\left(4 \mathrm{C}, 4 \mathrm{CH}_{2}\right), 114.88,127.55,137.72,147.25(4 \mathrm{C}$, thiophene carbons); [121.12 (2C), 125.83, 128.55 (2C), 128.99 (2C), 129.02 (2C), 129.51 (2C), 136.30, 12C, 2 $\left.\mathrm{C}_{6} \mathrm{H}_{5}\right], 157.81(\mathrm{C}=\mathrm{N}), 163.35(\mathrm{C}=\mathrm{O})$; $\mathrm{MS}: \mathrm{m} / z 437\left(\mathrm{M}^{+}, 88 \%\right), 421\left(\mathrm{M}^{+}-\mathrm{NH}_{2}, 18 \%\right)$, $345\left(\mathrm{M}^{+}-\left[\mathrm{CH}_{2} \mathrm{C}_{6} \mathrm{H}_{5}\right]+\mathrm{H}, 29 \%\right), 318\left(\mathrm{M}^{+}-\mathrm{CH}_{2} \mathrm{C}_{6} \mathrm{H}_{5}, \mathrm{HCN}+\mathrm{H}, 68 \%\right), 302\left(\mathrm{M}^{+}-\mathrm{CH}_{2} \mathrm{C}_{6} \mathrm{H}_{5}, \mathrm{HCN},-\mathrm{NH}_{2}\right.$, $+\mathrm{H}), 21 \%), 91\left(\mathrm{CH}_{2} \mathrm{C}_{6} \mathrm{H}_{5}, 100 \%\right)$.

\subsection{Synthesis of $\mathbf{3 0}, \mathbf{3 1}$}

A mixture of 26 (1.56 g, $5 \mathrm{mmol})$ and aromatic aldehyde $(5 \mathrm{mmol})$ in acetic acid $(30 \mathrm{~mL})$ was heated under reflux for $4 \mathrm{~h}$. Then the mixture was cooled and the solid separated was filtered, dried and recrystallized from a suitable solvent.

3-[N'-(3,4-Dimethoxybenzylidene)-hydrazino]-2-phenylamino-5, 6, 7,8-tetrahydro-3H-benzo[4,5] thieno[2,3-d]pyrimidin-4-one (30). Light yellow crystals, m.p. 192-194 ${ }^{\circ} \mathrm{C}$; Yield 56\%; IR $\left(\mathrm{cm}^{-1}\right)$ : 3,325 (NH), 3,053, 3,007, 1,672 (CO), 1,600, 1,570 (C=C); MS: $m / z 460\left(\mathrm{M}^{+}, 24 \%\right), 297\left(\mathrm{M}^{+}-\right.$ $\left.\left(\mathrm{N}=\mathrm{CHC}_{6} \mathrm{H}_{3}(\mathrm{OMe})_{2}\right)+\mathrm{H}, 44 \%\right), 180\left(\mathrm{M}^{+}-\left(\mathrm{N}=\mathrm{CHC}_{6} \mathrm{H}_{3}(\mathrm{OMe})_{2}, \mathrm{NHC}_{6} \mathrm{H}_{5}, \mathrm{HCN}\right)+2 \mathrm{H}, 16 \%\right)$.

3-[N'-(2,4-Dichlorobenzylidene)-hydrazino]-2-phenylamino-5, 6, 7,8-tetrahydro-3H-benzo[4,5]thieno [2,3-d] pyrimidin-4-one (31). Yellow powder, m.p. 192-194 ${ }^{\circ} \mathrm{C}$; Yield 51\%; IR $\left(\mathrm{cm}^{-1}\right): 3,319(\mathrm{NH})$, 3,068, 1,668 (CO), 1,598, 1,537, 1,544 (C=C); MS: $m / z 468\left(\mathrm{M}^{+}, 57 \%\right), 470\left(\mathrm{M}^{+}+2,33 \%\right), 323\left(\mathrm{M}^{+}-\right.$ $\left.\left(\mathrm{C}_{6} \mathrm{H}_{3} \mathrm{Cl}_{2}\right), 29 \%\right), 268\left(\mathrm{M}^{+}-\left(\mathrm{C}_{6} \mathrm{H}_{3} \mathrm{Cl}_{2}, \mathrm{HCN}, \mathrm{C}_{2} \mathrm{H}_{4}\right), 96 \%\right), 178\left(\mathrm{M}^{+}-\left(\mathrm{C}_{6} \mathrm{H}_{3} \mathrm{Cl}_{2}, \mathrm{HCN}, \mathrm{C}_{2} \mathrm{H}_{4}, \mathrm{NHC}_{6} \mathrm{H}_{5}+\right.\right.$ $2 \mathrm{H}, 46 \%)$.

\subsection{Synthesis of 4-amino-5,6-disubstituted[4,5]thieno[2,3-d]pyrimidines 33a,b}

A mixture of compound 32a,b $(10 \mathrm{mmol})$ and formamide $(10 \mathrm{~mL})$ was heated under reflux for $2 \mathrm{~h}$, then left to cool overnight at ambient temperature. The solid formed was filtered, dried and recrystallized from ethanol [24].

7-Benzyl-4-amino-5,6,7,8-tetrahydropyrido[3 ,4 :4,5]thieno[2,3-d]pyrimidine (33a). Light brown crystals, m.p. $236-237{ }^{\circ} \mathrm{C}$; yield: $73 \%$; IR $\left(\mathrm{cm}^{-1}\right)$ : 3,413, 3,313 $\left(\mathrm{NH}_{2}\right), 3,050,1,633,1,552(\mathrm{C}=\mathrm{C}) ;{ }^{1} \mathrm{H}-$ NMR (DMSO-d $\left.{ }_{6}\right) \delta: 2.77\left(2 \mathrm{H}, \mathrm{t}, \mathrm{CH}_{2}\right), 2.96\left(2 \mathrm{H}, \mathrm{t}, \mathrm{CH}_{2}\right), 3.62\left(2 \mathrm{H}, \mathrm{s}, \mathrm{CH}_{2}\right), 3.70\left(2 \mathrm{H}, \mathrm{s}, \mathrm{CH}_{2}\right), 6.85$ $\left(2 \mathrm{H}, \mathrm{s}, \mathrm{NH}_{2}\right), 7.27-7.36\left(5 \mathrm{H}, \mathrm{m}, \mathrm{C}_{6} \mathrm{H}_{5}\right), 8.18(1 \mathrm{H}, \mathrm{s}, \mathrm{H}-2) ;{ }^{13} \mathrm{C}-\mathrm{NMR}$ (DMSO-d 6 ) $\delta: 26.17,49.43$, 51.93, $61.16\left(4 \mathrm{C}, 4 \mathrm{CH}_{2}\right), 115.02,127.68,138.71,153.70$ (thiophene carbons), 126.13, 128.85 (2C), 129.13, 129.37, (2C) $\left(\mathrm{C}_{6} \mathrm{H}_{5}\right), 158.72$ (C-2), 166.30 (C-4); $\mathrm{MS}: m / z 296\left(\mathrm{M}^{+}, 25 \%\right), 205\left(\mathrm{M}^{+}-\left(\mathrm{CH}_{2^{-}}\right.\right.$ $\left.\left.\mathrm{C}_{6} \mathrm{H}_{5}\right), 50 \%\right), 177\left(\mathrm{M}^{+}-\left(\mathrm{CH}_{2}-\mathrm{C}_{6} \mathrm{H}_{5}, \mathrm{NCH}_{2}\right), 63 \%\right), 162\left(\mathrm{M}^{+}-\left(\mathrm{CH}_{2}-\mathrm{C}_{6} \mathrm{H}_{5}, \mathrm{NCH}_{2}, \mathrm{NH}_{2}\right)+\mathrm{H}, 2 \%\right)$. 
4-Amino-6,7,8,9-tetrahydro-5H-cyclohepta[4,5]thieno[2,3-d]pyrimidine (33b). Violet crystals, m.p. 264-266 ${ }^{\circ} \mathrm{C}$; Yield: 66\%; IR $\left(\mathrm{cm}^{-1}\right): 3,352,3,327\left(\mathrm{NH}_{2}\right), 3,143,1,651,1,556(\mathrm{C}=\mathrm{C})$; ${ }^{1} \mathrm{H}-\mathrm{NMR}$ $\left(\mathrm{DMSO}_{-} \mathrm{d}_{6}\right) \delta: 1.65\left(4 \mathrm{H}, \mathrm{m}, 2 \mathrm{CH}_{2}\right), 1.83\left(2 \mathrm{H}, \mathrm{m}, \mathrm{CH}_{2}\right) 2.83\left(2 \mathrm{H}, \mathrm{t}, \mathrm{CH}_{2}\right), 3.00\left(2 \mathrm{H}, \mathrm{t}, \mathrm{CH}_{2}\right), 6.92(2 \mathrm{H}$, $\left.\mathrm{s}, \mathrm{NH}_{2}\right), 8.15(1 \mathrm{H}, \mathrm{s}, \mathrm{H}-2)$; ${ }^{13} \mathrm{C}-\mathrm{NMR}\left(\mathrm{DMSO}_{6}\right) \delta: 26.98,27.40,29.11(2 \mathrm{C}), 31.34\left(5 \mathrm{C}, 5 \mathrm{CH}_{2}\right)$, 116.66, 132.68, 135.55, 152.96 (4C, thiophene carbons), 158.70 (C-2), 161.79 (C-4); MS: $m / z 219$ $\left(\mathrm{M}^{+}, 100 \%\right), 204\left(\mathrm{M}^{+}-\left(\mathrm{NH}_{2}\right)+\mathrm{H}, 23 \%\right), 190\left(\mathrm{M}^{+}-\left(\mathrm{NH}_{2}, \mathrm{CH}_{2}\right)+\mathrm{H}, 52 \%\right)$.

\subsection{5. (3-Cyano-5,6, 7,8-tetrahydro-4H-cyclohepta[b]thiophene-2-yl)-carbamic acid ethyl ester (34)}

A mixture of 32b (3.84 g, $20 \mathrm{mmol})$ and ethyl chloroformate $(2.3 \mathrm{~mL}, 24 \mathrm{mmol})$ in pyridine $(40 \mathrm{~mL})$, was stirred for $30 \mathrm{~h}$ at room temperature, the solvent was removed under reduced pressure to obtain a dark oil, treated with $3-5 \mathrm{~mL}$ of water, the the precipitate collected by filtration, dried and recrystallized from ethanol to give violet crystals of 34, m.p. $135-137^{\circ} \mathrm{C}$; Yield: $49 \%$; IR $\left(\mathrm{cm}^{-1}\right)$ : 3,215 (NH), 2,223 (CN), 1,724 (CO), 1,571, 1,556 (C=C), 1,240 (C-O); ${ }^{1} \mathrm{H}-\mathrm{NMR}\left(\mathrm{CDCl}_{3}\right) \delta: 1.33(3 \mathrm{H}$, t, $\left.J=7.32 \mathrm{~Hz}, \mathrm{CH}_{3}\right), 1.64\left(4 \mathrm{H}, \mathrm{m}, 2 \mathrm{CH}_{2}\right), 1.82\left(2 \mathrm{H}, \mathrm{m}, \mathrm{CH}_{2}\right), 2.67\left(4 \mathrm{H}, \mathrm{m}, 2 \mathrm{CH}_{2}\right), 4.26(2 \mathrm{H}, \mathrm{q}$, $\left.J=7.32 \mathrm{~Hz}, \mathrm{CH}_{2}-\mathrm{O}\right), 7.69(1 \mathrm{H}, \mathrm{s}, \mathrm{NH}) ;{ }^{13} \mathrm{C}-\mathrm{NMR}\left(\mathrm{CDCl}_{3}\right) \delta: 14.44\left(\mathrm{CH}_{3}\right), 27.34,28.06,29.13,29.22$, $32.04\left(5 \mathrm{C}, 5 \mathrm{CH}_{2}\right), 62.87\left(\mathrm{CH}_{2}-\mathrm{O}\right), 95.30,130.98,136.04,146.13$ (4C, thiophene carbons), 114.81 $(\mathrm{CN}), 152.67(\mathrm{C}=\mathrm{O})$.

\subsection{2,3,5,7,8,9,10,11-Octahydro-[1,3]-imidazolo[2,3-c]-4-oxo-cyclohepta[4,5]thieno[2,3- d]pyrimidine (35)}

Ethylenediamine $(1 \mathrm{~mL})$ was added dropwise with stirring to a solution of compound 34 (264 $\mathrm{mg}$, $1 \mathrm{mmol})$ in DMF $(5 \mathrm{~mL})$ within $2 \mathrm{~h}$ at $150{ }^{\circ} \mathrm{C}$. After cooling, water $(20 \mathrm{~mL})$ was added to the solution and the precipitated crystals were collected by filtration, washed with water, dried and crystallized from ethanol to give colorless crystals, m.p. $275-276{ }^{\circ} \mathrm{C}$; yield: $80 \%$; IR $\left(\mathrm{cm}^{-1}\right): 3,138(\mathrm{NH}), 1,643$ (CO), 1,573, 1,525 (C=C); ${ }^{1} \mathrm{H}-\mathrm{NMR}\left(\mathrm{CDCl}_{3}\right) \delta: 1.52\left(4 \mathrm{H}, \mathrm{m}, 2 \mathrm{CH}_{2}\right), 1.69\left(2 \mathrm{H}, \mathrm{m}, \mathrm{CH}_{2}\right), 2.53$ $\left(2 \mathrm{H}, \mathrm{t}, J=5.8 \mathrm{~Hz}, \mathrm{CH}_{2}\right), 2.94\left(2 \mathrm{H}, \mathrm{t}, J=5.8 \mathrm{~Hz}, \mathrm{CH}_{2}\right), 3.79\left(2 \mathrm{H}, \mathrm{m}, \mathrm{N}-\mathrm{CH}_{2}\right), 3.90\left(2 \mathrm{H}, \mathrm{m}, \mathrm{N}-\mathrm{CH}_{2}\right)$, $2.44(1 \mathrm{H}, \mathrm{s}, \mathrm{NH}) ;{ }^{13} \mathrm{C}-\mathrm{NMR}\left(\mathrm{CDCl}_{3}\right) \delta: 24.17,24.85,25.10,26.31,29.09\left(5 \mathrm{C}, 5 \mathrm{CH}_{2}\right), 40.04(2 \mathrm{C}$, $\left.2 \mathrm{CH}_{2}-\mathrm{N}\right), 112.00,121.50,127.35,132.11$ (thiophene carbons), $146.21(\mathrm{CN}), 149.52(\mathrm{C}=\mathrm{O}) ; \mathrm{MS}: \mathrm{m} / \mathrm{z}$ $261\left(\mathrm{M}^{+}, 100 \%\right), 220\left(\mathrm{M}^{+}-\left(\mathrm{NCH}_{2} \mathrm{CH}_{2}\right)+\mathrm{H}, 50 \%\right)$.

\subsection{7-Benzyl-4-imino-3-amino-5, 6, 7,8-tetrahydro-4H-pyrido[3 '4 $: 4,5]$ thieno $[2,3-d]$ pyrimidine (36)}

A mixture of 32a $(20 \mathrm{mmol})$ and triethylorthoformate $(20 \mathrm{~mL})$ was heated under reflux for $4 \mathrm{~h}$, and the excess of the reagent was then removed under vacuum, A mixture of hydrazine hydrate $(99 \%$, $5 \mathrm{~mL}$ ) and ethanol $(15 \mathrm{~mL})$ was added to the residue with stirring, and it was allowed to stand overnight at room temperature. The solid obtained was filtered, washed with ethanol, dried and crystallized from ethanol. Fine brown crystals, m.p. 166-167 ${ }^{\circ} \mathrm{C}$; Yield: $67 \%$; IR $\left(\mathrm{cm}^{-1}\right) ; 3,354,3,253$, 3,174 ( $\left.\mathrm{NH}_{2}, \mathrm{NH}\right), 3,094-3,031(\mathrm{Ar}-\mathrm{CH}), 1,652,1,606(\mathrm{C}=\mathrm{N}), 1,554,1,541(\mathrm{C}=\mathrm{C}) ;{ }^{1} \mathrm{H}-\mathrm{NMR}\left(\mathrm{CDCl}_{3}\right)$ $\delta: 2.98\left(2 \mathrm{H}, \mathrm{t}, \mathrm{CH}_{2}\right), 3.21\left(2 \mathrm{H}, \mathrm{s}, \mathrm{CH}_{2}\right), 3.72\left(4 \mathrm{H}, \mathrm{m}, 2 \mathrm{CH}_{2}\right), 5.42\left(2 \mathrm{H}, \mathrm{s}, \mathrm{NH}_{2}\right), 7.25-7.37(5 \mathrm{H}, \mathrm{m}$, $\left.\mathrm{C}_{6} \mathrm{H}_{5}\right), 8.08(\mathrm{H}, \mathrm{s}, \mathrm{CH}), 8.87(1 \mathrm{H}, \mathrm{s}, \mathrm{NH})$. 
7-Benzyl-4-imino-3-amino-2-methyl-5,6,7,8-tetrahydro-4H-pyrido[3 ,4 :4,5] thieno[2,3-d] pyrimidine (37). Prepared from 32a $(20 \mathrm{mmol})$ and triethyl orthoacetate $(20 \mathrm{~mL})$ following the same procedure as for 36 above. Fine light brown crystals, m.p. $172-174{ }^{\circ} \mathrm{C}$; Yield : 54\%; IR $\left(\mathrm{cm}^{-1}\right): 3,330,3,284,3,178$ $\left(\mathrm{NH}_{2}, \mathrm{NH}\right), 3,094-3,028(\mathrm{Ar}-\mathrm{CH}), 1,652,1,610(\mathrm{C}=\mathrm{N}), 1,544,1,521(\mathrm{C}=\mathrm{C}) ;{ }^{1} \mathrm{H}-\mathrm{NMR}\left(\mathrm{CDCl}_{3}\right) \delta: 2.79$ $\left(3 \mathrm{H}, \mathrm{s}, \mathrm{CH}_{3}\right), 2.83\left(2 \mathrm{H}, \mathrm{t}, J=5.8 \mathrm{~Hz}, \mathrm{CH}_{2}\right), 2.97\left(2 \mathrm{H}, \mathrm{t}, J=5.8 \mathrm{~Hz}, \mathrm{CH}_{2}\right), 3.58\left(2 \mathrm{H}, \mathrm{s}, \mathrm{CH}_{2}\right), 3.69(2 \mathrm{H}$, $\left.\mathrm{s}, \mathrm{CH}_{2}\right), 6.14\left(2 \mathrm{H}, \mathrm{br}, \mathrm{s}, \mathrm{NH}_{2}\right), 7.34-7.28\left(6 \mathrm{H}, \mathrm{m}, \mathrm{NH}, \mathrm{C}_{6} \mathrm{H}_{5}\right) ;{ }^{13} \mathrm{C}-\mathrm{NMR}\left(\mathrm{CDCl}_{3}\right)$ \&: $22.75\left(\mathrm{CH}_{3}\right)$, 26.18, 49.43, 51.70, $61.94\left(4 \mathrm{C}, 4 \mathrm{CH}_{2}\right), 115.59,127.18,137.53,154.59$ (thiophene carbons), 127.50, 128.57 (2C), 128.18 (2C), $133.00\left(6 \mathrm{C}, \mathrm{C}_{6} \mathrm{H}_{5}\right), 156.05$ (C-2), 160.50 (C-4).

4-Imino-3-amino-6, 7,8,9-tetrahydro-4H,5H-cyclohepta[4,5]thieno[2,3-d]pyrimidine (38). Prepared from 32b $(20 \mathrm{mmol})$ with triethyl orthoformate $(20 \mathrm{~mL})$ following the procedure above described for 36. Fine colorless crystals, m.p: $164-165^{\circ} \mathrm{C}$; yield: $56 \%$; IR $\left(\mathrm{cm}^{-1}\right): 3,294,3,286,3,163\left(\mathrm{NH}_{2}, \mathrm{NH}\right)$, 3,053 (Ar-CH), 1,639, 1,614 (C=N), 1,562 (C=C); ${ }^{1} \mathrm{H}$ NMR $\left(\mathrm{CDCl}_{3}\right)$ 8: $1.79\left(4 \mathrm{H}, \mathrm{m}, 2 \mathrm{CH}_{2}\right), 1.91(2 \mathrm{H}$, $\left.\mathrm{m}, \mathrm{CH}_{2}\right), 2.74\left(2 \mathrm{H}, \mathrm{m}, \mathrm{CH}_{2}\right), 3.06\left(2 \mathrm{H}, \mathrm{m}, \mathrm{CH}_{2}\right), 4.75\left(2 \mathrm{H}\right.$, brs, $\left.\mathrm{NH}_{2}\right), \mathrm{NH}$ not observed, $7.96(1 \mathrm{H}, \mathrm{s}$, $\left.\mathrm{C}^{2}-\mathrm{H}\right) ;{ }^{13} \mathrm{C}-\mathrm{NMR}\left(\mathrm{CDCl}_{3}\right)$ \&: 25.53, 27.34, 29.23, 29.36, $31.20\left(5 \mathrm{C}, 5 \mathrm{CH}_{2}\right), 120.44,135.02,138.08$, 146.41 (4C, thiophene carbons), 155.31 (C-2), 155.99 (C-4).

4-Imino-3-amino-2-methyl-6,7,8,9-tetrahydro-4H,5H-cyclohepta[4,5]thieno[2,3-d] pyrimidine (39). Prepared from 32b $(20 \mathrm{mmol})$ with triethyl orthoacetate $(20 \mathrm{~mL})$ following the procedure of $\mathbf{3 6}$. Fine colorless crystals, m.p. $186-188^{\circ} \mathrm{C}$; Yield: 70\%; IR $\left(\mathrm{cm}^{-1}\right)$; 3,354, 3,284, 3,190 $\left(\mathrm{NH}_{2}, \mathrm{NH}\right), 3,001$ (Ar$\mathrm{CH}), 1,647,1,606(\mathrm{C}=\mathrm{N}), 1,552,1,521(\mathrm{C}=\mathrm{C}) ;{ }^{1} \mathrm{H}-\mathrm{NMR}\left(\mathrm{CDCl}_{3}\right) \delta: 1.71\left(4 \mathrm{H}, \mathrm{m}, 2 \mathrm{CH}_{2}\right), 1.83(2 \mathrm{H}, \mathrm{m}$, $\left.\mathrm{CH}_{2}\right), 2.67\left(3 \mathrm{H}, \mathrm{s}, \mathrm{CH}_{3}\right), 2.77\left(2 \mathrm{H}, \mathrm{m}, \mathrm{CH}_{2}\right), 3.00\left(2 \mathrm{H}, \mathrm{m}, \mathrm{CH}_{2}\right), 5.50\left(2 \mathrm{H}, \mathrm{br}, \mathrm{s}, \mathrm{NH}_{2}\right), \mathrm{NH}$ not observed; ${ }^{13} \mathrm{C}-\mathrm{NMR}\left(\mathrm{CDCl}_{3}\right) \delta: 22.53\left(1 \mathrm{C}, \mathrm{CH}_{3}\right), 26.41,27.21,29.14,29.25,30.96\left(5 \mathrm{C}, 5 \mathrm{CH}_{2}\right)$, $117.51,134.13,138.23,154.94$ (thiophene ring), 155.55 (C-2), 157.28 (C-4).

3.28. 8-Benzyl-2-methyl-7,8,9,10-tetrahydropyrido[4 '3`-4,5] thieno[3,2-e][1,2,4]triazolo[2,3c]pyrimidine (40)

Compound 36 (10 mmol) was heated under reflux for $2 \mathrm{~h}$ with an excess of triethyl orthoacetate $(20 \mathrm{~mL})$ and the excess of the reagent was then removed under vacuum. The solid obtained was washed with ethanol, dried and crystallized from ethanol to give brown crystals, m.p. 157-159 ${ }^{\circ} \mathrm{C}$; Yield: 45\%; IR $\left(\mathrm{cm}^{-1}\right)$ : 3,041 (Ar-CH), 1,618 (C=N), 1,556, 1,508 (C=C); ${ }^{1} \mathrm{H}-\mathrm{NMR}\left(\mathrm{CDCl}_{3}\right) \delta: 2.65$ $\left(3 \mathrm{H}, \mathrm{s}, \mathrm{CH}_{3}\right), 2.99\left(2 \mathrm{H}, \mathrm{m}, \mathrm{CH}_{2}\right), 3.27\left(2 \mathrm{H}, \mathrm{m}, \mathrm{CH}_{2}\right), 3.79\left(4 \mathrm{H}, \mathrm{s}, 2 \mathrm{CH}_{2}\right), 7.34-7.38\left(5 \mathrm{H}, \mathrm{m}, \mathrm{C}_{6} \mathrm{H}_{5}\right)$, $9.06(1 \mathrm{H}, \mathrm{s}, \mathrm{CH}) ;{ }^{13} \mathrm{C}-\mathrm{NMR}\left(\mathrm{CDCl}_{3}\right) \delta: 14.78\left(\mathrm{CH}_{3}\right), 25.62,49.79,51.77,61.91\left(4 \mathrm{C}, 4 \mathrm{CH}_{2}\right), 119.65$, 127.43, 136.20, 137.79 (thiophene carbons), 127.52, $128.55(2 \mathrm{C}), 129.12(2 \mathrm{C}), 135.44\left(6 \mathrm{C}, \mathrm{C}_{6} \mathrm{H}_{5}\right)$, $149.29(\mathrm{C}=\mathrm{N}), 154.06(\mathrm{C}=\mathrm{N}), 165.25(\mathrm{C}=\mathrm{N})$; $\mathrm{MS}: \mathrm{m} / z 335\left(\mathrm{M}^{+}, 11 \%\right), 244\left(\mathrm{M}^{+}-\left(\mathrm{CH}_{2}-\mathrm{C}_{6} \mathrm{H}_{5}\right), 10 \%\right)$, $216\left(\mathrm{M}^{+}-\left(\mathrm{CH}_{2}-\mathrm{C}_{6} \mathrm{H}_{5}, \mathrm{NCH}_{2}\right), 37 \%\right)$.

8-Benzyl-4-methyl-7,8,9,10-tetrahydro-pyrido[4`3 -4,5]thieno[3,2-e][1,2,4]triazolo[2,3-c]pyrimidine (41). Prepared from $37(10 \mathrm{mmol})$ and triethyl orthoformate $(20 \mathrm{~mL})$ following the procedure given for 40. Fine light yellow scales, m.p. $142-144{ }^{\circ} \mathrm{C}$; Yield 39\%; IR $\left(\mathrm{cm}^{-1}\right) ; 3,084-3,060$ (Ar-CH), 1,652, 1,622 (C=N), 1,558, 1,517 (C=C); ${ }^{1} \mathrm{H}-\mathrm{NMR}\left(\mathrm{DMSO}_{-} \mathrm{d}_{6}\right) \delta: 2.97\left(3 \mathrm{H}, \mathrm{s}, \mathrm{CH}_{3}\right), 3.04\left(2 \mathrm{H}, \mathrm{m}, \mathrm{CH}_{2}\right), 3.30$ 
$\left(2 \mathrm{H}, \mathrm{br}, \mathrm{s}, \mathrm{CH}_{2}\right), 3.82\left(4 \mathrm{H}, \mathrm{m}, 2 \mathrm{CH}_{2}\right), 7.26-7.40\left(5 \mathrm{H}, \mathrm{m}, \mathrm{C}_{6} \mathrm{H}_{5}\right), 8.36(1 \mathrm{H}, \mathrm{s}, \mathrm{CH}) ; \mathrm{MS}: \mathrm{m} / z 335\left(\mathrm{M}^{+}\right.$, $8 \%), 244\left(\mathrm{M}^{+}-\left(\mathrm{CH}_{2}-\mathrm{C}_{6} \mathrm{H}_{5}\right), 30 \%\right), 216\left(\mathrm{M}^{+}-\left(\mathrm{CH}_{2}-\mathrm{C}_{6} \mathrm{H}_{5}, \mathrm{NCH}_{2}\right), 100 \%\right)$.

4-Methyl-8,9,10,11-tetrahydro-7H-cyclohepta[4,5]thieno[3,2-e][1,2,4]triazolo[2,3-c]pyrimidine (42). Prepared from $39(10 \mathrm{mmol})$ and triethyl orthoformate $(20 \mathrm{~mL})$ following the procedure given above for 40. Fine colorless crystals, m.p. $159-160{ }^{\circ} \mathrm{C}$; Yield: $67 \%$; IR $\left(\mathrm{cm}^{-1}\right): 3,089(\mathrm{Ar}-\mathrm{CH}), 1,618(\mathrm{C}=\mathrm{N})$, 1,550, 1,521 (C=C); ${ }^{1} \mathrm{H}-\mathrm{NMR}\left(\mathrm{CDCl}_{3}\right) \delta: 1.74\left(4 \mathrm{H}, \mathrm{m}, 2 \mathrm{CH}_{2}\right), 1.92\left(2 \mathrm{H}, \mathrm{m}, \mathrm{CH}_{2}\right), 2.91\left(2 \mathrm{H}, \mathrm{m}, \mathrm{CH}_{2}\right)$, $2.95\left(3 \mathrm{H}, \mathrm{s}, \mathrm{CH}_{3}\right), 3,41\left(2 \mathrm{H}, \mathrm{m}, \mathrm{CH}_{2}\right), 8.34(1 \mathrm{H}, \mathrm{s}, \mathrm{C}-\mathrm{H}) ;{ }^{13} \mathrm{C}-\mathrm{NMR}\left(\mathrm{CDCl}_{3}\right) \delta: 19.64\left(\mathrm{CH}_{3}\right), 27.34$, 27.85, 28.41, 30.46,32.42 (5C, 5 $\left.\mathrm{CH}_{2}\right), 120.55,134.11,141.56,145.43$ (4C, thiophene carbons), 149.04 $(\mathrm{C}=\mathrm{N}), 152.01(\mathrm{C}=\mathrm{N}), 153.32(\mathrm{C}=\mathrm{N})$; MS: $m / z 258\left(\mathrm{M}^{+}, 100 \%\right), 243\left(\mathrm{M}^{+}-\left(\mathrm{CH}_{3}\right), 37 \%\right), 230\left(\mathrm{M}^{+}-\right.$ $\left.\left(\mathrm{C}_{2} \mathrm{H}_{4}\right), 69 \%\right), 216\left(\mathrm{M}^{+}-\left(\mathrm{C}_{2} \mathrm{H}_{4}, \mathrm{CH}_{3}\right)+\mathrm{H}, 18 \%\right)$.

\section{4,2-Dimethyl-8,9,10,11-tetrahydro-7H-cyclohepta[4,5]thieno[3,2-e][1,2,4]triazolo[2,3-c]pyrimidine}

(43). Prepared from $39(10 \mathrm{mmol})$ and triethyl orthoacetate $(20 \mathrm{~mL})$ following the procedure described above for 40, Fine colorless crystals, m.p. $189-190{ }^{\circ} \mathrm{C}$; Yield: $64 \%$; IR $\left(\mathrm{cm}^{-1}\right)$ : 2,922-2,854 (aliphatic $\mathrm{CH}), 1,618(\mathrm{C}=\mathrm{N}), 1,550,1,519(\mathrm{C}=\mathrm{C}) .{ }^{1} \mathrm{H}-\mathrm{NMR}\left(\mathrm{CDCl}_{3}\right) \delta: 1.77\left(4 \mathrm{H}, \mathrm{m}, 2 \mathrm{CH}_{2}\right), 1.93\left(2 \mathrm{H}, \mathrm{m}, \mathrm{CH}_{2}\right)$, $2.64\left(3 \mathrm{H}, \mathrm{s}, \mathrm{CH}_{3}\right), 2.94\left(5 \mathrm{H}, \mathrm{CH}_{2}, \mathrm{CH}_{3}\right), 3,42\left(2 \mathrm{H}, \mathrm{m}, \mathrm{CH}_{2}\right) .{ }^{13} \mathrm{C}-\mathrm{NMR}\left(\mathrm{CDCl}_{3}\right) \delta: 14.63\left(\mathrm{CH}_{3}\right), 19.64$ $\left(\mathrm{CH}_{3}\right), 27.3,27.8,28.37,30.4,32.1\left(5 \mathrm{C}, 5 \mathrm{CH}_{2}\right), 119.89,133.89,140.71,151.96$ (thiophene carbons), 149.51 (C-4), 144.88 (C-10), 163.47 (C-2); MS: m/z $272\left(\mathrm{M}^{+}, 82 \%\right), 243\left(\mathrm{M}^{+}-\left(2 \mathrm{CH}_{3}\right)+\mathrm{H}, 60 \%\right)$, $230\left(\mathrm{M}^{+}-\left(\mathrm{CH}_{3}, \mathrm{C}_{2} \mathrm{H}_{4}\right)+\mathrm{H}, 47 \%\right)$.

3.29. 8-Benzyl-7-8,9,10-tetrahydro-3H-pyrido[4 ,3 :4,5] thieno[3,2-e][1,2,4]triazolo[2,3c]pyrimidine-2-thione (44)

A mixture of $36(1 \mathrm{mmol})$ in pyridine $(10 \mathrm{~mL})$ and carbon disulfide $(400 \mathrm{mg}, 5 \mathrm{mmol})$ was heated under reflux for $3 \mathrm{~h}$. The solid obtained after cooling, was collected and recrystallized from ethanol. Fine orange scales, m.p. 237-238 ${ }^{\circ} \mathrm{C}$; Yield: $36 \%$; IR $\left(\mathrm{cm}^{-1}\right): 3,379(\mathrm{NH}) 3,028(\mathrm{Ar}-\mathrm{CH}), 2,850-2,920$ (aliphatic $\mathrm{CH}), 1,525,1,454(\mathrm{C}=\mathrm{C}), 1,392(\mathrm{C}=\mathrm{S}) ;{ }^{1} \mathrm{H}-\mathrm{NMR}\left(\mathrm{CDCl}_{3}\right)$ 8:2.87 $\left(2 \mathrm{H}, \mathrm{m}, \mathrm{CH}_{2}\right), 3.68(2 \mathrm{H}, \mathrm{m}$, $\left.\mathrm{CH}_{2}\right), 4.01\left(4 \mathrm{H}, \mathrm{m}, 2 \mathrm{CH}_{2}\right), 7.50-7.52(3 \mathrm{H}, \mathrm{m}), 7.41-7.68(2 \mathrm{H}, \mathrm{m}), 8.14(1 \mathrm{H}, \mathrm{s}, \mathrm{H}-2)$.

3.30. 8,9,10,11-Tetrahydro-3H,7H-cyclohepta[4,5] thieno[3,2-e][1,2,4]triazolo[2,3-c]pyrimidine-2thione (45)

A mixture of $38(1 \mathrm{mmol})$ in pyridine $(10 \mathrm{~mL})$ and carbon disulfide $(400 \mathrm{mg}, 5 \mathrm{mmol})$ was heated under reflux for $3 \mathrm{~h}$. Work up following the procedure given above for $\mathbf{4 4}$ and recrystallization from ethanol gave 45 as yellow scales, m.p. 275-276 ${ }^{\circ} \mathrm{C}$; Yield: $75 \%$; IR $\left(\mathrm{cm}^{-1}\right)$ : 3,057 (Ar-CH), 2,9845-2,999 (aliphatic $\mathrm{CH}), 1,517,1,454(\mathrm{C}=\mathrm{C}), 1,365(\mathrm{C}=\mathrm{S}) .{ }^{1} \mathrm{H}-\mathrm{NMR}\left(\mathrm{CDCl}_{3}\right) \delta: 1.70-2.0(6 \mathrm{H}, \mathrm{m}$, $\left.3 \mathrm{CH}_{2}\right), 2.98\left(4 \mathrm{H}, \mathrm{m}, 2 \mathrm{CH}_{2}\right), 9.43(1 \mathrm{H}, \mathrm{s}, \mathrm{NH}) ;{ }^{13} \mathrm{C}-\mathrm{NMR}\left(\mathrm{CDCl}_{3}\right) \delta: 26.56,26.96,28.99,30.65,31.38$ $\left(5 \mathrm{C}, 5 \mathrm{CH}_{2}\right), 125.21,132.25,149.53,153.5$ (4C, thiophene carbons), 135.65 (C-2), 157.67 (C-4), $182.39(\mathrm{C}=\mathrm{S})$. 
4-Methyl-8,9,10,11-tetrahydro-3H,7H-cyclohepta[4,5] thieno[3,2-e][1,2,4]triazolo[2,3-c]pyrimidine2-thione (46). Prepared from 38 (1 mmol) in pyridine $(10 \mathrm{~mL})$ and carbon disulfide (400 $\mathrm{mg}, 5 \mathrm{mmol})$ following procedure described for 45. Yellow scales, m.p. $288-290{ }^{\circ} \mathrm{C}$; Yield: $80 \%$; IR $\left(\mathrm{cm}^{-1}\right): 2,916$, 2,846 (aliphatic $\left.\mathrm{CH}_{2}\right), 1,618(\mathrm{C}=\mathrm{N}), 1,550,1,519(\mathrm{C}=\mathrm{C}) ;{ }^{1} \mathrm{H}-\mathrm{NMR}\left(\mathrm{CDCl}_{3}\right) \delta: 1.77\left(4 \mathrm{H}, \mathrm{m}, 2 \mathrm{CH}_{2}\right)$, $1.93\left(2 \mathrm{H}, \mathrm{m}, \mathrm{CH}_{2}\right), 2.91\left(2 \mathrm{H}, \mathrm{m}, \mathrm{CH}_{2}\right), 2.98\left(2 \mathrm{H}, \mathrm{m}, \mathrm{CH}_{2}\right), 3.03\left(3 \mathrm{H}, \mathrm{s}, \mathrm{CH}_{3}\right) ;{ }^{13} \mathrm{C}-\mathrm{NMR}\left(\mathrm{CDCl}_{3}\right) \delta$ : $22.37\left(1 \mathrm{C}, \mathrm{CH}_{3}\right), 26.52,26.96,28.57,30.35,31.24\left(5 \mathrm{C}, 5 \mathrm{CH}_{2}\right), 123.90,131.68,146.95,153.68(4 \mathrm{C}$, thiophene carbons), $153.68(\mathrm{C}-2), 157.94(\mathrm{C}-4), 181.21(\mathrm{C}=\mathrm{S})$.

\section{Conclusions}

Several new thienopyrimidin-4-one(thione) derivatives were synthesized from 2-amino-3carbethoxy (or 3-cyano)-4,5-disubstituted thiophenes as starting materials. Further, some triazolothienopyrimidine and 2-thioxothienopyrimidine representatives have also been synthesized, and their structures have been determined on the basis of their 2D NMR data. Some of prepared compounds were evaluated for their antimicrobial and antitumor -activities, and compounds 4a, 12, 15 were found to be promising antimicrobial agents, while compounds $7 \mathbf{a}, \mathbf{8 a}, \mathbf{1 5}, \mathbf{2 9}, \mathbf{4 2}$ have some activity against different human tumor cell lines.

\section{Acknowledgments}

One of the authors, Khulud Al-Tisan, is grateful to the College of Science at Dammam University and the College of Science at King Fisal University in Al-Ahasa, for financial support and providing all facilities for this research as part of her Ph.D thesis. The authors are thankful to KACST for support, and to the Chemistry Department at King Saud University for running all the NMR analyses. We are indebted to Abdul Aziz Al-Masaud, Ali Al-assayed, at King Abdul Aziz Hospital for the National Guard at Al-Ahsa for supplying the antibiotics and providing the facilities for the antimicrobial tests in this research.

\section{References}

1. Quintela, J.; Peinador, C.; Moreira, M.; Alfonso, A.; Botana, L.; Riguera, R. Pyrazolopyrimidines: synthesie, effect on histamine release from rat peritoneal mast cells and cytotoxic activity. Eur. $J$. Med. Chem. 2001, 36, 321-332.

2. Heyman, F.R.; Bousquer, P.; Cunha, G.; Moskey, M.; Ahmed, A.; Soni, N.; Marcotte, P.; Pease, P.; Glaser, K.; Yates, M.; Bouska, J.; Albert, D.; Blak-Schaefer, D.P.; Stewart, K.; Rafferty, P.; Davidsen, S.; Curtin, M. Thienopyrimidine urea inhibitors of KDR kinase. Bioorg. Med. Chem. Lett. 2007, 17, 1246-1249.

3. Ashalatha, B.V.; Narayana, B.; Vijaya raj, K.K.; Kumari, S. Synthesis of some new bioactive 3amino-2-mercabto -5, 6, 7, 8- tetrahydro [1] benzothieno [2,3-d] pyrimidin-4-one derivatives. Eur. J. Med. Chem. 2007, 42, 719-728.

4. Katada, J.; Lijima, K.; Muramatsu, M.; Takami, M.; Yasuda, E.; hayashi, M.; Hattori, M.; Hayashi, Y. Cytotoxic effects of NSL-1406, anew thienopyrimidine derivative, on leukocytes and osteoclasts. Bioorg. Med. Chem. Lett. 1999, 9, 797-802. 
5. K.Jain, K.; Bariwal, J.; Kathiravan, M.; Phoujdar, M.; Sahne, R.; Chauhan, B.; Shah, A.; Yadav, M. Recent advances in selective $\alpha_{1}$-adrenoreceptor antagonists as antihypertensive agents. Bioorg. Med. Chem. Lett. 2008, 16, 4759-4800.

6. Gewald, K. Heterocycles from $\mathrm{CH}$-acidic nitriles. VII 2 aminothiophene from a-oxo mercaptans and methylene-active nitriles. Chem. Ber. 1965, 98, 3571-3577.

7. Alagarsamy, V.; Vijayakumar, S.; Solomon, V.R. Synthesis of 2-mercapto-3-substituted-5,6dimethylthieno[2,3-d]pyrimidin-4(3H)-ones as new analgesic, anti-inflammatory agents. Biomed. Phamacol. 2007, 61, 285-291.

8. Bhutyan, M.; Rahman, M.; Abdurrahim, K.; Hossain, M.; Abunaser, M. Synthesis and anti microbial evaluation of some new thienopyrimidine derivatives. Acta Pharm. 2006, 56, 411-450.

9. El-Sherbeny, M.A.; El-Ashmawy, M.B.; El-Subbagh, H.I.; El-Emam, A.A.; Synthesis, antimicrobial and antiviral evaluation of certain thienopyrimidine derivatives. Eur. J. Med. Chem. 1995, 30, 445-449.

10. Alagarsamy, V.; Meena, S.; Ramseshu, K.V.; Solomon, V.R.; Thirumuruganb, K.; Dhanabala, K.; Murugan, M. Synthesis, analgesic, anti-inflammatory, ucerogenic index and antibacterial activities of novel 2-methylthio-3-substituted-5,6,7,8-tetrahydrobenzo (b) thieno[2,3-d]pyrimidin4-(3H)-ones. Eur. J. Med. Chem. 2006, 41, 1293-1300.

11. El-Shafei, A.; Fadda, A.A.; Khalil, A.M.; Ameen. T.A.E.; Badria, F.A. Synthesis, antitumor evaluation, molecular modeling and quantitative structure-activity relationship (QSAR) of some novel arylazopyrazolodiazine and triazine analogs. Bioorg. Med. Chem. 2009, 17, 5096-5105.

12. El-Baih, F.E.M; Al-Taisan, K.M.; AL-Hazimi, H.M. Synthesis of Some new substituted Thieno[2,3-d]pyrimidines and related heterocyclic systems. J. Stat. Comput. Simul. 2000, 4, 281-290.

13. El-Baih, F.E.M.; Al-Blowy, H.A.S.; AL-Hazimi, H.M. Synthesis of Some Thienopyrimidine Derivatives. Molecules 2006, 11, 498-513.

14. Gewald, K.; Schinke, E.; Bottcher. H. 2-Amin-thiophene aus methylenakteven Nitrilen, Carbonylverbindungen und Schwefel. Chem. Ber. 1966, 99, 94-100.

15. Elslager, E.; Jacob, P.; Werbel, L. Folate Antagonists. 6. Synthesis and Antimalarial Effects of Fused 2,4-Diaminothieno[2,3-d] pyrimidine(1-3). J. Heterocycl. Chem. 1972, 11, 775-782.

16. Murray, P.R.; Baron, E.J. Jorgensen, J.H.; Pfaller, M.A.; Yulken, R.H. Manual of Clinical Microbiology; American Society for Microbiology Press (ASM): Washington, DC, USA, 2003; p. 1212.

17. Delost, M.D. Introduction to Diagnostics Microbiology, Text and Work Book; Mosby, Inc.: Sc, Louis, MO, USA, 1997; p. 552.

18. Skehan P. New colorimetric cytotoxicity assay for anti-cancer drug screening. J. Natl. Cancer Inst. 1990, 82, 1107-1112.

19. Lambert, J.B.; Mazzola, E.P. NMR Spectroscopy, an Introduction to Principles, Applications, and Experimental Methods; Pearson Education 1td.: London, UK, 2004; p. 341.

20. Pavia, D.L.; Lampman, G.M.; Kriz, G.S., Jr. Introduction to Spectroscopy, 4th ed.; Brooks Cole: Belrnont, CA, USA, 2009; p. 656. 
21. Modica, M.; Santagati, M.; Guccione, S.; Russo, F.; Cagnotto, A.; Goega, M.; Mennini, T. Design, Synthesis and binding properties of novel and selective 5- $\mathrm{HT}_{3}$ and $5-\mathrm{HT}_{4}$ receptor ligands. Eur. J. Med. Chem. 2000, 35,1065-1079.

22. Ahmed, E.Kh.; Sensfuss, U.; Habicher, W.D. Fusions of Pyrido[4',3:4,5]thieno[2,3-d] pyrimidines with N-Heterocyclic Moieties. J. Heterocycl. Chem. 1999, 36, 1119-1122.

23. EL-Saghier, A.M. A Simple Synthesis of Some New Thienopyridine and Thienopyrimidine Derivatives. Molecules 2002, 7, 756-766.

Sample Availability: Samples of the compounds are available from the authors.

(C) 2010 by the authors; licensee MDPI, Basel, Switzerland. This article is an Open Access article distributed under the terms and conditions of the Creative Commons Attribution license (http://creativecommons.org/licenses/by/3.0/). 